Edith Cowan University

Research Online

Research outputs 2014 to 2021

$12-1-2017$

\title{
Establishing the theoretical components of alexithymia via factor analysis: Introduction and validation of the attention-appraisal model of alexithymia
}

\author{
David Preece \\ Edith Cowan University \\ Rodrigo Becerra \\ Edith Cowan University \\ Alfred Allan \\ Edith Cowan University \\ Ken Robinson \\ Edith Cowan University \\ Justine Dandy \\ Edith Cowan University
}

Follow this and additional works at: https://ro.ecu.edu.au/ecuworkspost2013

Part of the Psychology Commons

10.1016/j.paid.2017.08.003

This is an Author's Accepted Manuscript of: Preece, D., Becerra, R., Allan, A., Robinson, K., \& Dandy, J. (2017).

Establishing the theoretical components of alexithymia via factor analysis: Introduction and validation of the attention-appraisal model of alexithymia. Personality and Individual Differences, 119, 341-352.

https://doi.org/10.1016/j.paid.2017.08.003

This Journal Article is posted at Research Online.

https://ro.ecu.edu.au/ecuworkspost2013/3247 
Establishing the Theoretical Components of Alexithymia via Factor Analysis: Introduction and Validation of the Attention-Appraisal Model of Alexithymia

David A. Preece ${ }^{1}$, Rodrigo Becerra ${ }^{2}$, Alfred Allan ${ }^{1}$, Ken Robinson $^{1}$, Justine Dandy ${ }^{1}$

${ }^{1}$ Edith Cowan University, Perth, Australia.

${ }^{2}$ The University of Western Australia, Perth, Australia. 


\begin{abstract}
Alexithymia is an important mental health construct, but there is continuing debate regarding its definition and measurement. We attempt to resolve this definitional uncertainty in two ways. Firstly, we trace the development of the alexithymia construct, focusing particularly on what we call the Toronto and Amsterdam models, and examine a body of empirical research that shows strong support for the hypothesis that alexithymia consists of three components (difficulty identifying feelings, difficulty describing feelings, and externally orientated thinking). Based on these components, we formulate an alternate theoretical model of alexithymia, the attention-appraisal model of alexithymia, that aligns alexithymia theory with recent advances in the broader emotion regulation field. Secondly, we examine the construct's latent structure by factor analysing data from multiple psychometric measures administered to a community sample $(N=368)$. Our results suggest statistical support for our model, rather than the Toronto or Amsterdam models. We end by discussing how our model accounts for several unresolved issues within the alexithymia field, including the construct's relation to imaginal capacities and emotional reactivity, whether alexithymia is a deficit or a defence, how it might be addressed in psychiatric treatment, and the discordance that has existed between alexithymia theory and alexithymia measurement.
\end{abstract}


Establishing the Theoretical Components of Alexithymia via Factor Analysis: Introduction and Validation of the Attention-Appraisal Model of Alexithymia

Psychoanalytic practitioners working with psychosomatic patients in the middle part of the 20th century observed that they often presented with a cluster of emotion ${ }^{7}$ processing deficits. These patients were unable to "describe their feelings or to differentiate among them" and displayed "an absence of the capacity to produce fantasies with the result that [their] thought content [was] restricted to a preoccupation with external objects, people, and environmental events" (Nemiah, 1984, p. 127). Sifneos and Nemiah first used the term alexithymia (from the Greek, $a=$ lack, lexis = word, thymos $=$ feeling) to describe this phenomenon (Nemiah \& Sifneos, 1970; Sifneos, 1973).

Modern authors still use the term alexithymia to describe this cluster of emotion processing deficits and it is widely considered to be a dimensional trait that is normally distributed in the general population (e.g., Parker, Keefer, Taylor, \& Bagby 2008).

Researchers have further confirmed that psychosomatic patients do typically present with elevated levels of alexithymia (Duddu, Isaac, \& Chaturvedi, 2003) and it is also regarded as an important transdiagnostic risk factor for a range of psychopathologies, including, depression (Honkalampi, Hintikka, Laukkanen, \& Viinamaki, 2001), anxiety disorders (Zeitlin \& McNally, 1993), personality disorders (Berenbaum, 1996), eating disorders (Taylor et al., 1996), and substance use (Thorberg et al., 2009). The alexithymia construct is, thus, of substantial clinical interest. There is, however, continuing debate regarding its definition, measurement, and theoretical underpinnings (Lane et al., 2015; Taylor et al., 2016; Watters, Taylor, \& Bagby, 2016).

\footnotetext{
${ }^{7}$ Most authors (e.g., Gross, 2014; Lane \& Schwartz, 1987) use the term emotion to refer to loosely coupled changes that arise across three channels of the emotion system: the subjective-experiential (e.g., feeling of fear), physiological (e.g., increased heart rate), and behavioural (e.g., urge to run) channels. This is the meaning we intend when using the term emotion throughout this paper.
} 
In this paper, we attempt to resolve some of this definitional uncertainty in two ways. Firstly, we trace the development of the alexithymia construct, focusing particularly on what we will refer to as the Toronto and Amsterdam models, and examine a body of empirical research that shows strong support for the hypothesis that alexithymia consists of three components. Based on these components, we use Gross's (2015a) extended process model of emotion regulation to formulate an alternate theoretical model of alexithymia that we refer to as the attention-appraisal model of alexithymia. Secondly, we conduct a study whereby we factor analyse multiple psychometric measures in order to examine the latent structure of the alexithymia construct, and in so doing, test some important predictions of our model.

\section{Developmental history and empirical research}

The dominant contemporary work in developing the alexithymia construct has been done by two groups of researchers who we will refer to as the Toronto and Amsterdam groups. These two groups have proposed different definitions of alexithymia, and almost all contemporary researchers select between these definitions when describing the construct. Below, we outline each of these models and detail the results of empirical work that has tested their specifications.

Toronto model. The Toronto group (Taylor et al., 1999) developed a model of alexithymia building on the work of pioneers in the area (Marty \& M'Uzan, 1963; Nemiah, 1977; Nemiah \& Sifneos, 1970; Sifneos, 1973) who used psychoanalytic concepts to explain their observations. Marty and de M'Uzan (1963), for example, proposed that alexithymia was due to disturbance in the early child-mother relationship that disrupted childhood development of the ability to experience feelings or use fantasy as a means to satisfy instinctual drives. Proponents of this psychoanalytical approach, therefore, believed that people with high levels of alexithymia were prone to experiencing somatic symptoms because they were unable to use fantasy and psychic elaboration to regulate the energy of 
their instinctual drives (see also, McDougall, 1974; Nemiah, 1977).

The Toronto group built on this psychoanalytic theorising by also applying cognitive theories of emotion processing (e.g., Bucci's [1997] multiple code theory, and Lane and Schwartz's [1987] cognitive-developmental theory of levels of emotional awareness) to the understanding of alexithymia, whilst still retaining the multidimensional structure of the construct originally described by Nemiah and Sifneos (1970). The Toronto model, therefore, specifies that alexithymia is comprised of four interrelated (positively correlated) components: difficulty identifying feelings in the self (DIF); difficulty describing feelings (DDF); an externally orientated thinking (EOT) style whereby one tends to focus excessively on the details of the external world rather than focusing attention on their internal states; and constricted imaginal processes (difficulty fantasising; DFAN), marked by the absence or scarcity of daydreams and fantasies. The Toronto model further specifies that the DIF and DDF components are closely linked to form a broader affect awareness component, and the EOT and DFAN components form a broader operative thinking component (see Figure 5.1) (Bagby et al., 2006; Taylor et al., 1999). This model is presently the most widely used definition of alexithymia within the literature (Watters, Taylor, Quilty, \& Bagby, 2016).

The Toronto group developed two measures of alexithymia based on their model, the 20-item Toronto Alexithymia Scale (TAS-20; Bagby et al., 1994) and the Toronto Structured Interview for Alexithymia (TSIA; Bagby et al., 2006). The TSIA is an observer-rated measure with items designed to measure DIF, DDF, EOT and DFAN, and the TAS-20 is a self-report questionnaire with items designed to measure DIF, DDF and EOT. The earliest version of TAS-20, known as the TAS (Taylor et al., 1985), also included DFAN items, however the Toronto group removed the DFAN items in later revisions (Taylor Bagby, \& Parker, 1992).

Empirical support. Subsequent empirical work using these measures has allowed for 
the latent structure of the alexithymia construct to be tested. This work has supported much of the Toronto model. Examinations of the TAS-20's psychometric structure consistently find that scores on the DIF, DDF and EOT subscales correlate significantly and positively, and load on the same higher-order factor in factor analysis (e.g., Bagby et al., 1994; Gignac et al., 2007; Meganck et al., 2008). Similarly, to our knowledge, in all studies the DIF, DDF and EOT subscales of the TISA correlate positively (Bagby et al., 2006; Caretti et al., 2011; Grabe et al., 2009; Inslegers et al., 2013), suggesting that these components could be part of the same latent construct. The Toronto model's specification that DFAN is part of the same construct as DIF, DDF and EOT has, however, garnered less psychometric support.

To the best of our knowledge, all psychometric studies using the original TAS have found that the DFAN subscale is uncorrelated or negatively correlated with the DIF and DDF subscales (e.g., Taylor et al., 1985; Haviland, Hendryx, Cummings, Shaw, \& MacMurray 1991); the Toronto group cite these results as a reason the DFAN subscale was removed from revisions of the measure (Taylor et al., 1992). Conversely, all studies using the TSIA have found that its DFAN subscale does correlate coherently (positively) with its DIF, DDF and EOT subscales (Bagby et al., 2006; Caretti et al., 2011; Grabe et al., 2009; Inslegers et al., 2013), however, cross-correlations with other alexithymia measures suggest that this may primarily be due to shared method variance (Podsakoff et al., 2003). Namely, of the five samples where correlations between the TSIA and TAS-20 have been examined (Bagby et al., 2006; Caretti et al., 2011; Grabe et al., 2009; Inslegers et al., 2013), in most (three) samples, the TSIA DFAN subscale did not correlate significantly with the TAS-20 total scale score (Bagby et al., 2006; Caretti et al., 2011), and in Caretti et al. (2011) the TSIA DFAN subscale was negatively correlated with the TAS-20 DIF subscale. Similarly, we know of one study (Rosenberg et al., 2016) that has compared the TSIA to the Bermond-Vorst Alexithymia Questionnaire (BVAQ; Vorst \& Bermond, 2001), and in this case, the TSIA 
DFAN subscale correlated strongly with the BVAQ DFAN subscale, but did not correlate with the BVAQ DIF, DDF or EOT subscales. Indeed, in the one study to conduct a network analysis of the TSIA, Watters, Taylor and Bagby (2016) found that the DFAN items did not fit well within the same network as the DIF, DDF and EOT items. Most experimental studies have documented similar findings. In most experimental work, people with high or low levels of alexithymia (grouped based on TAS-20 total scale scores) are found to not differ with respect to imaginal efficiency (Czernecka \& Szymura, 2008) or the vividness of visual imagery (Bausch et al., 2011; Golena, 2014; Mantani, Okamoto, Shirao, Okada, \& Yamawaki, 2005; but see Campos, Chiva, \& Moreau, 2000; Friedlander, Lumley, Farchione, \& Doyal, 1997). This collection of empirical findings has, consequently, led some authors to recently question the extent to which DFAN is a feature of alexithymia (e.g., Bausch et al., 2011; Morera, Culhane,Watson, \& Skewes, 2005; Watters, Taylor, \& Bagby, 2016; Watters, Taylor, Quilty, \& Bagby, 2016).

Amsterdam model. The Amsterdam group (Vorst \& Bermond, 2001) propose an alternate definition of alexithymia. The Amsterdam group built on the four components of alexithymia delineated by the Toronto group (DIF, DDF, EOT, DFAN) and added a fifth component, reduced emotional reactivity (or difficulty emotionalising; DEMO). Vorst and Bermond (2001, p. 417) define emotional reactivity as "the degree to which someone is emotionally aroused by emotion inducing events", which is a narrower definition than is typically used within the emotional reactivity field (see Becerra \& Campitelli, 2013), but raises the possibility that alexithymic people do not experience emotions (i.e., along the subjective-experiential channel of the emotion system) as intensely as other people. The Amsterdam group justify the inclusion of these five components within their definition of alexithymia based on their interpretation that they were described by Nemiah and Sifneos (1970). The Amsterdam group, further, organise these five components according to a 
different higher-order structure, whereby they are subsumed within two broader components that are orthogonal to each other; cognitive alexithymia, composed of DIF, DDF and EOT, and affective alexithymia, composed of DFAN and DEMO (see Figure 5.1). Vorst and Bermond (2001) specify that there are subtypes of alexithymia; type I alexithymia, where people have difficulties in both cognitive alexithymia and affective alexithymia, and type II alexithymia, where people have difficulties only in cognitive alexithymia.

The Amsterdam group developed a self-report measure of alexithymia based on their model, the aforementioned BVAQ (Vorst \& Bermond, 2001), which includes items designed to assess DIF, DDF, EOT, DFAN and DEMO. Bermond et al. (1999) originally specified that these five components should be positively correlated, thus, the separation of these components into an orthogonal structure seems, in our interpretation of their work, to be psychometrically driven.

Empirical support. Psychometric studies of the BVAQ have found that it conforms to this orthogonal structure in factor analyses. The DIF, DDF and EOT subscales load together onto a higher-order factor (cognitive alexithymia), and the DFAN and DEMO subscales load on a separate higher-order factor (affective alexithymia); these higher-order factors are largely uncorrelated (e.g., Bermond et al., 2007; Vorst \& Bermond, 2001; Zech, Luminet, Rimé, \& Wagner, 1999). Factor analytic work with the BVAQ has therefore been consistent with the Amsterdam model, and the body of TAS-20 work described earlier can also be seen as supportive of the cognitive alexithymia construct. Other empirical work has, however, been inconsistent with some specifications of the Amsterdam model.

We are aware of only one cluster analysis study that has examined whether the type I and type II alexithymia subtypes exist statistically, and in this case (using BVAQ data), no such subtypes were found (Bagby et al., 2009). Moreover, with respect to the inclusion of DEMO within the Amsterdam model, most empirical work not using the BVAQ DEMO 
subscale to operationalise emotional reactivity has found that cognitive alexithymia is not orthogonal to emotional reactivity. Namely, most experimental studies have found that individuals with high DIF, DDF and EOT report feeling significantly higher levels of negative affect in response to stressful stimuli or laboratory tasks (e.g., Eastabrook et al., 2013; Connelly \& Denney, 2007; Newton \& Contrada, 1994; Pollatos et al., 2011; but see Luminet, Rimé, Bagby, \& Taylor, 2004), and elevated levels of cognitive alexithymia are commonly present in clinical groups whose symptoms are characterised by hyper-reactivity within the emotion system (e.g., borderline personality disorder; New et al., 2012). Studies using psychometric measures of psychological distress or personality, similarly, find that cognitive alexithymia is associated with significantly higher levels of self-reported negative affect (e.g., Baily \& Henry, 2007; Leising, Grande, \& Faber, 2009; Li, Zhang, Guo, \& Zhang, 2015; Lundh \& Simonsson-Sarnecki, 2001; Morera et al., 2005) and neuroticism (a personality trait characterised by emotional instability and proneness to negative affect; e.g., Muller et al., 2004; Morera et al., 2005).

We think these inconsistencies between the BVAQ literature and other literature with respect to DEMO might be accounted for by the manner in which the BVAQ operationalises DEMO. Earlier, we noted that Vorst and Bermond's (2001) definition of emotional reactivity is narrower than that typically used in the emotional reactivity field. In that field, the construct is usually defined as the ease of activation, intensity and duration of one's emotional responses, and a distinction is further made between negative reactivity (reactivity with negative emotions) and positive reactivity (reactivity with positive emotions) (Becerra et al., 2017; Becerra \& Campitelli, 2013; Davidson, 1998). Emotional reactivity researchers consider the distinction between negative reactivity and positive reactivity to be of particular import, as statistically they are separate dimensions that are negatively correlated with each other (Becerra et al., 2017). Vorst and Bermond (2001), however, do not make a distinction 
between positive and negative reactivity in their definition, and this is reflected their BVAQ DEMO subscale; four items refer to negative emotions, one refers to positive emotions, and three do not specify a valence. In our view, not specifying valence in a DEMO item may be problematic as the respondent must guess what type of emotion was meant, and the combining of negative and positive valence items into a single score is not theoretically supported due to the negative correlation between these reactivity dimensions (see Becerra et al., 2017). In our view, three of the BVAQ DEMO items also appear to measure empathy rather than emotional reactivity. These three items are specific to whether an emotion is elicited by compassion or care for others (e.g., item 9 "When I see somebody crying uncontrollably, I remain unmoved"), and are therefore inseparable from the personal distress facet of empathy delineated by Davis (1983) in his popular model of empathy. Empathy, as a construct, is negatively correlated with alexithymia (Grynberg, Luminet, Corneille, Grèzes, \& Berthoz., 2010), and most authors consider empathy and emotional reactivity to be separable constructs (Becerra \& Campitelli, 2013; Davidson, 1998). The BVAQ DEMO items have, indeed, been found to be inconsistent in the direction of their correlations with other constructs (Watters, Taylor, Quilty, \& Bagby, 2016) and the subscale has displayed low internal consistency in some samples (e.g., Muller et al., 2004). We, consequently, place more weight in the findings of those studies that did not use the BVAQ DEMO subscale to operationalise emotional reactivity, and most of these findings indicate that cognitive alexithymia is not orthogonal to emotional reactivity.

Summary of empirical findings. The weight of the empirical literature, therefore, appears to support most (but not all) specifications of the Toronto and Amsterdam models. Both models agree that DIF, DDF and EOT are interrelated components of a common latent construct, and on this point there is strong empirical support. The weight of the empirical literature, however, suggests that some parts of these models may be misspecified, in that 
DFAN and DEMO do not, statistically, appear to be part of this same construct. The attention-appraisal model of alexithymia

The empirical findings summarised above suggest that the alexithymia construct might be better defined as consisting of only DIF, DDF and EOT. To provide an empirically credible framework for future research and explain why only these three components statistically cohere, we hence propose a new theoretical model of alexithymia; the attentionappraisal model of alexithymia. In formulating this model, we also use this opportunity to incorporate recent advances in the broader emotion regulation field (Gross, 2015a) into alexithymia theory, because we believe these advances might provide a useful theoretical framework for conceptualising alexithymia. We specifically use Gross's (2015a) recently introduced extended process model of emotion regulation as a framework for our model of alexithymia, because many authors consider it to be at the forefront of emotion regulation theory and it has been successfully applied to a multitude of emotional phenomena (e.g., Aldao \& Christensen, 2015; Diaz \& Eisenberg, 2015; Giuliani \& Berkman, 2015; Kuppens \& Verduyn, 2015; Schmader \& Mendes, 2015). Within this framework, we also seek to integrate Lane and Schwartz's (1987) cognitive-developmental theory of levels of emotional awareness. Lane and Schwartz's theory has been discussed and accepted previously by the Toronto group (Taylor et al., 1999), but we think integrating it within Gross's (2015a) model will provide some more clarity.

The core tenet of Gross's (2015a) extended process model of emotion regulation is that people generate, process, and regulate emotions via valuation systems. All valuation systems are comprised of a four stage situation-attention-appraisal-response ${ }^{8}$ sequence,

\footnotetext{
${ }^{8}$ In Gross's (2015a) extended process model of emotion regulation, these stages are actually labelled the worldperception-valuation-action stages, respectively. We, instead, use the original labels for these stages (i.e., the labels used in the earlier modal model of emotion [Gross, 1998] and process model of emotion regulation [Gross, 1998]) because we consider these alternate labels to be more intuitively descriptive for the purpose of conceptualising alexithymia.
} 
whereby a stimulus is valuated (evaluated) in terms of its meaning for the individual. Emotions are considered to be generated via a valuation system where: an emotion inducing stimulus is present (situation stage; e.g., a snake is in the room), the individual focuses their attention on the stimulus (attention stage; e.g., looking at the snake), they appraise the stimulus in terms of what it is and what it means for their goals (appraisal stage; e.g., this snake in the room is bad for the goal of staying alive), and an emotional response results (response stage; e.g., fear). This emotional response can itself then become the focus of valuation, whereby it is valuated in terms of whether it is a desired state. Specifically, to valuate an emotional response (situation stage) the individual must focus attention on the emotion (attention stage), appraise the emotion in terms of what it is and what it means for their goals (appraisal stage), and they then might activate a goal to engage in action that reduces the discrepancy between the current state of the world and their desired state of the world (response stage; e.g., the feeling of fear is not a desired state so the individual runs away from the snake in an attempt to reduce the feeling). The response stage of this latter valuation system represents emotion regulation within Gross's (2015b, p. 130) model, defined as "the activation of a goal to modify an unfolding emotional response".

In the attention-appraisal model of alexithymia, we propose that it is within this same valuation system that alexithymia manifests. We posit that EOT can be conceptualised as difficulty at the attention stage of this valuation system. That is, an emotional response has occurred, but the individual has difficulty focusing their attention on it. Similarly, DIF and DDF can be conceptualised as difficulties at the appraisal stage of this valuation system. That is, an emotional response has occurred, but the individual has difficulty accurately appraising what the emotional response is and what it means. There is a subtle shift in emphasis here when describing EOT relative to early descriptions from psychoanalytic commentators (e.g., Nemiah, 1984); the pertinent point is not that the alexithymic individual 
focuses excessively on external objects or events, but rather, from the reverse perspective, that they do not properly focus their attention on their emotions. Consequently, whilst we agree with the close clustering of DIF and DDF within the Toronto model, we propose difficulty appraising as a label for this grouping rather than affect awareness, because we think EOT also represents an affect awareness problem (one at the attention stage of emotion valuation). Alexithymia is, therefore, conceptualised as a closely clustered set of difficulties during the emotion valuation process described by Gross (2015a).

The degree of difficulty people experience at the attention and appraisal stages of emotion valuation, we believe, can be understood in terms of the levels of emotional awareness specified by Lane and Schwartz (1987). Based on Piaget's (1981) theory of cognitive development, Lane and Schwartz delineate five developmental levels (or stages); ranging from level one and two, where the individual can experience emotions only as global bodily sensations or diffuse unpleasant/pleasant states, to levels three, four and five, where the perception of discrete and specific emotions becomes possible and gradually more nuanced. Lane and Schwartz posit that people's level of emotional awareness depends on the degree of development in their emotion schemas, which Lane and Schwartz define as those cognitive structures that guide the processing of emotions. Based on interpersonal interactions, people's emotion schemas are thought to develop and become hierarchically organised, more complex, integrated, and differentiated. Experimental researchers have, indeed, since found evidence suggestive of impaired emotion schema functioning in people with high levels of alexithymia (e.g., Lane et al., 1996; Luminet, Vermeulen, Demaret, Taylor, \& Bagby, 2006; Lundh, Johnsson, Sundqvist, \& Olsson, 2002; Suslow \& Junghanns, 2002; Vermeulen, Luminet, \& Corneille, 2006).

We, therefore, follow Lane and Schwartz's (1987) theorising when positing in our model that difficulties at the attention and appraisal stages of emotion valuation can occur 
due to what we call ability deficit alexithymia, that is, people's emotion schemas being underdeveloped (i.e., poorly organised, differentiated, and integrated). People's schemas guide the manner in which they attend to the world (e.g., Markus, 1977) and if their emotion schemas are underdeveloped, they may be unable to focus on the most pertinent aspects of the emotional response during the attention stage of emotion valuation. People also use their schemas during the appraisal stage, and if these emotion schemas are underdeveloped they may be unable to accurately, or with sufficient degrees of differentiation, interpret patterns of input information about the emotion to enable valuation at a specific (e.g., "I am feeling embarrassed" or "I am feeling angry"), rather than a broad or diffuse (e.g., "I am feeling bad"), level (Lane \& Schwartz, 1987).

Ability deficit alexithymia by itself, however, fails to explain why some people's overall levels of alexithymia often increase during periods of distress (e.g., Luminet, Bagby, \& Taylor, 2001; Luminet, Rokbani, Ogez, \& Jadoulle, 2007), or recent findings that the association between alexithymia and psychiatric symptoms is sometimes mediated by experiential avoidance (e.g., Bilotta, Giacomantonio, Leone, Mancini, \& Coriale, 2015; Panayiotou et al., 2015). Recent findings, thus, suggest that some difficulties at the attention and appraisal stages of emotion valuation may also be caused by what we call avoidance alexithymia. We hypothesise that avoidance alexithymia represents an avoidant emotion regulation strategy (attentional deployment; see Gross, 2014) whereby at the attention stage, people may avoid properly focusing their attention on the emotional response, and at the appraisal stage, people may avoid linking available input information about the stimulus (i.e., the emotion) to their emotion schemas. As we illustrate in Figure 5.2, in our model, avoidance alexithymia is activated at the response (i.e., emotion regulation) stage of a valuation system that is valuating an emotion, but the result of this avoidance response then ultimately manifests at the attention and appraisal stages of subsequent valuation systems; 
that is, the avoidance behaviour that is activated as an emotion regulation strategy causes the individual to regress to operating at a lower developmental level at the attention and appraisal stages of subsequent emotion processing.

In sum, alexithymia is conceptualised as a set of difficulties during the attention (EOT) and appraisal (DIF, DDF) stages of emotion valuation. The overall extent of these difficulties is determined by the developmental level of people's emotion schemas (i.e., ability deficit alexithymia) and the degree to which they are using experiential avoidance of emotions as a regulation strategy (i.e., avoidance alexithymia).

Toronto model

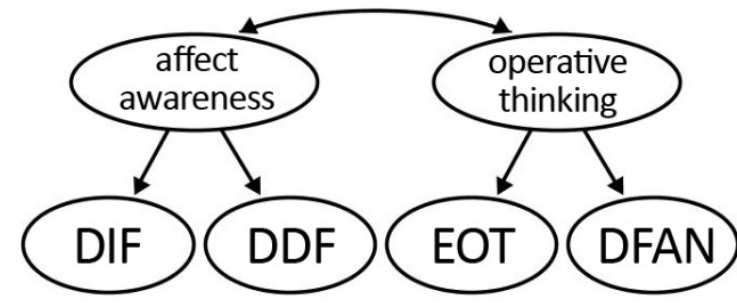

Amsterdam model

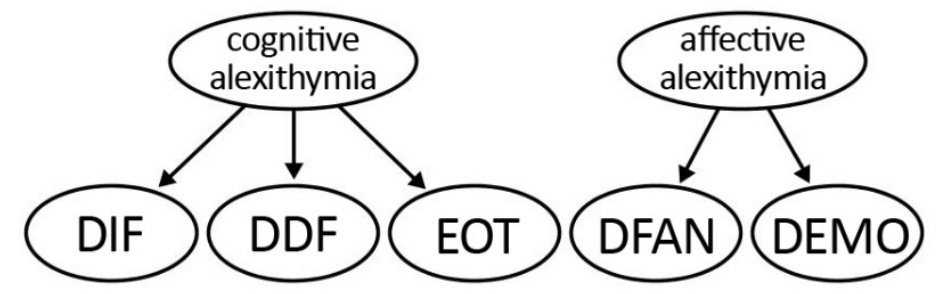

attention-appraisal model

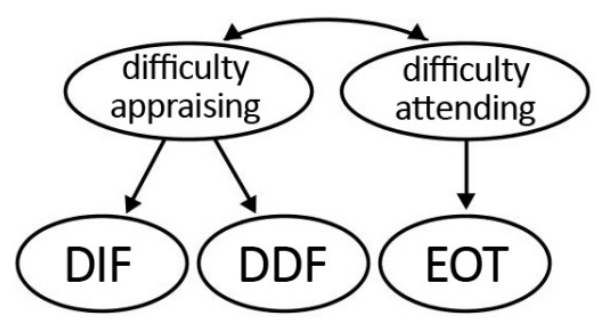

Figure 5.1. A visual representation of the different theoretical models that attempt to describe the structure of the alexithymia construct; the Toronto model, the Amsterdam model, and the attentionappraisal model. Double headed arrows indicate that the constructs are conceptualised as being positively correlated. DIF = difficulty identifying feelings, DDF = difficulty describing feelings, EOT $=$ externally orientated thinking, DFAN = difficulty fantasising, $\mathrm{DEMO}=$ difficulty emotionalising (low emotional reactivity). 


\section{Valuation system 1: emotion generation}

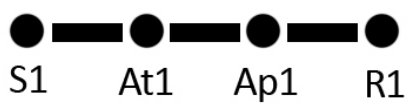

\section{Valuation system 2: emotion processing}

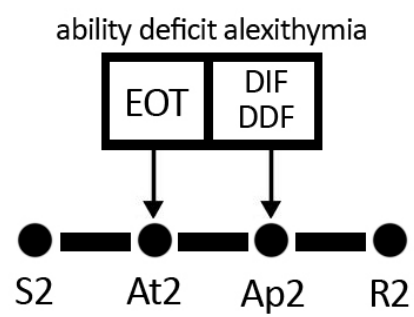

\section{Valuation system 3: subsequent emotion processing}

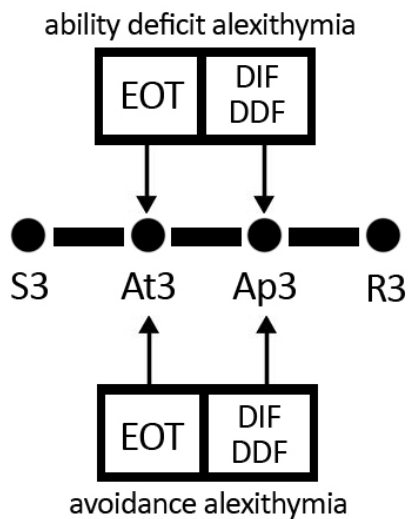

Figure 5.2. A visual representation of where, according to the attention-appraisal model of alexithymia, alexithymia manifests during the emotion valuation process. EOT = externally orientated thinking, DIF = difficulty identifying feelings, DDF = difficulty describing feelings. All valuation systems are comprised of four sequential stages; a situation (S), attention (At), appraisal (Ap) and response (R) stage (Gross, 1998, 2015a). Valuation system 1 represents an emotion being generated, whereby an emotion inducing stimulus is present (S1), attention is focused on the stimulus (At1), the stimulus is appraised in terms of what it is and what it means for the individual's goals (Ap1), and an emotional response results (R1). In valuation system 2, this emotional response can itself become the stimulus that is the target of valuation (S2), whereby attention is focused on the emotion (At2), it is appraised in terms of what it is and whether it is a desired state (Ap2), and then a goal might be activated to modify the unfolding emotional response (R2, i.e., emotion regulation). Some degree of ability deficit alexithymia (whether that be a high or low amount of difficulties) is present in all valuation systems with an emotion at the $\mathrm{S}$ stage, and manifests as difficulties at the At (EOT) and Ap (DIF, DDF) stages. Such difficulties reflect the developmental level of one's emotion schemas. Valuation system 3 represents subsequent emotion processing, after valuation system 2 has finished. In valuation system 3, the individual might have additional difficulties at the At3 and Ap3 stages attributable to avoidance alexithymia. These additional difficulties will occur if, in the R2 stage of valuation system 2 , the individual activated a goal to modify the emotion (i.e., an emotion regulation strategy) by using experiential avoidance; that is, attempting to avoid focusing attention on (EOT) or appraising (DIF, DDF) the emotional response in subsequent emotion processing. 


\section{Statistical comparison of the available models}

The attention-appraisal model, Toronto model, and Amsterdam model all make testable and different predictions about the latent structure of the alexithymia construct. Whilst we consider the existing body of psychometric work to be consistent with the notion that alexithymia is comprised of three components, we also think that some further work is needed before a three component definition is accepted. Specifically, existing studies have factor analysed single measures in isolation, and as a result, whilst informative, their results are more vulnerable to being influenced by the intricacies of that specific test. In this study, we therefore seek to advance the literature base by administering multiple self-report measures of alexithymia and emotional reactivity, and using exploratory and confirmatory factor analyses to examine the latent structures common to all measures. Our research question being: is the latent structure of the alexithymia construct, when assessed via selfreport measures, consistent with the attention-appraisal model, Toronto model, or Amsterdam model?

\section{Method}

\section{Participants and procedure}

Our data-set was comprised of 368 English speaking adults (209 females) from the general community. All participants were living in Australia at the time of the study, and their age ranged from 18 to $83(M=49.56, S D=16.67)$. For $31.6 \%$ their highest level of completed education was high school, for $35.6 \%$ it was a technical diploma, and for $32.6 \%$ it was a university degree. Most of the sample (90.8\%) were not currently studying at university. Participants were recruited via an online survey recruiting company (Qualtrics panels) or an advertisement placed on a social media website. Participants completed a battery of self-report questionnaires administered via an online anonymous survey. Some additional participants (recruited in the same manner) also completed the questionnaire 
battery, but their data were excluded during screening because they failed at least one of three attention check questions and/or completed the survey impossibly quickly (suggesting inattentive responding).

\section{Materials}

Included in the questionnaire battery were four questionnaires designed to measure alexithymia or emotional reactivity. Internal reliability coefficients for all administered measures are displayed in Table 5.1.

Toronto Alexithymia Scale-20. The TAS-20 (Bagby et al., 1994) is a 20 item selfreport measure of alexithymia. Items correspond to three subscales representing DIF (T-DIF subscale; 7 items, e.g., "I am often confused about what emotion I am feeling”), DDF (TDDF subscale; 5 items, e.g., "It is difficult for me to find the right words for my feelings") and EOT (T-EOT subscale; 8 items, e.g., "Being in touch with emotions is essential" [reverse-scored]). These subscales are combined together into a TOTAL SCALE score representing overall levels of alexithymia. Because this total score only includes items assessing DIF, DDF and EOT, it is consistent with alexithymia as it is specified by the attention-appraisal model. Participants respond to each item on a 5-point Likert scale ranging from 1 (strongly disagree) to 5 (strongly Agree), with higher scores indicating greater levels of alexithymia. Research has supported the validity and reliability of most scores derived from the TAS-20, though the T-EOT subscale typically has low internal reliability (Kooiman et al., 2002; Meganck et al., 2008).

Bermond-Vorst Alexithymia Questionnaire. The BVAQ (Vorst \& Bermond, 2001) is a 40 item self-report measure of alexithymia. Items are designed to measure DIF (DIDENTIFYING subscale; 8 items, e.g., "When I am upset, I know whether I am afraid or sad or angry" [reverse-scored]), DDF (D-VERBALSING subscale; 8 items, e.g., "I find it difficult to express my feelings verbally"), EOT (D-ANALYSING subscale; 8 items, e.g., "I 
hardly ever consider my feelings"), DFAN (D-FANTASISING subscale; 8 items, e.g., "I have few daydreams and fantasies"), and DEMO (D-EMOTIONALISING subscale; 8 items, e.g., "When friends around me argue violently, I become emotional" [reverse-scored]). Standard scoring involves calculating subscale scores for these five facets. The DIDENTIFYING, D-VERBALISING and D-ANALYSING subscales are also combined together into a COGNITIVE ALEXITHYMIA score, and the D-FANTASISING and DEMOTIONALISING subscales are combined together into an AFFECTIVE ALEXITHYMIA score. The COGNITIVE ALEXITHYMIA score is consistent with alexithymia as it is specified by the attention-appraisal model. Participants respond to each item on a 5-point scale ranging from 1 (this in no way applies) to 5 (this definitely applies). Higher scores indicate greater levels of alexithymia (for the D-EMOTIONALISING subscale, higher scores indicate lower levels of emotional reactivity). The validity and reliability of most scores derived from the BVAQ have been supported (Vorst \& Bermond, 2001; Bermond et al., 2007), though as noted earlier, we have some concerns about the construct validity of the D-EMOTIONALISING subscale.

Difficulties in Emotion Regulation Scale. The Difficulties in Emotion Regulation Scale (DERS; Gratz \& Roemer, 2004) is a 36 item self-report measure of emotion regulation and alexithymia. Two of its subscales conceptually correspond to the DIF and EOT facets of alexithymia. The CLARITY subscale (5 items, e.g., "I have no idea how I'm feeling”) is a measure of DIF, and the AWARENESS subscale (6 items, e.g., "I pay attention to how I feel" [reverse-scored]) is a measure of EOT. The DERS also includes four other subscales that measure aspects of emotion regulation. Participants respond to each item on a 5-point scale ranging from 1 (almost never) to 5 (almost always), with higher scores indicating greater difficulties. For this study, we combined the CLARITY and AWARENESS subscales together to form an ALEXITHYMIA COMPOSITE score. This ALEXITHYMIA 
COMPOSITE score is consistent with alexithymia as it is specified by the attention-appraisal model, though incomplete in that it does not include the DDF aspect of appraisal. The DERS subscales have demonstrated good validity and reliability (Gratz \& Roemer, 2004).

Perth Emotional Reactivity Scale. The PERS (Becerra et al., 2017) is a 30 item selfreport measure of emotional reactivity. It measures the typical ease of activation, intensity, and duration of one's emotional responses, and does so for negative and positive emotions separately. Six subscales can be derived from the PERS, each with 5 items: POSITIVEACTIVATION (e.g., "I tend to get happy very easily"), POSITIVE-INTENSITY (e.g., "When I am joyful, I tend to feel it very deeply"), POSITIVE-DURATION (e.g., "When I'm feeling positive, I can stay like that for a good part of the day"), NEGATIVE-ACTIVATION (e.g., "I tend to get upset very easily"), NEGATIVE-INTENSITY (e.g., "If I'm upset, I feel it more intensely than everyone else"), and NEGATIVE-DURATION (e.g., "Once in a negative mood, it's hard to snap out of it"). The three subscales within each valence domain can also be combined together into a GENERAL POSITIVE REACTIVITY scale score or GENERAL NEGATIVE REACTIVITY scale score, representing overall levels of reactivity for that valence. Items are answered on a 5-point Likert scale ranging from 1 (very unlike me) to 5 (very like me), with higher scores indicating higher levels of reactivity. The PERS has demonstrated good validity and reliability (Becerra et al., 2017).

\section{Analytic strategy}

Confirmatory factor analyses (CFAs) were conducted using AMOS 24, all other analyses were conducted using SPSS 24.

Pearson correlations and exploratory factor analysis. Pearson correlations between all administered scales/subscales were calculated; to control for type 1 error given the large number of correlations, a Bonferroni corrected alpha level of $<.001$ was used as the criteria for statistical significance. Subscale scores from the measures were used to perform an 
exploratory factor analysis (EFA; principal axis factoring with direct oblimin rotation).

Confirmatory factor analyses. Subscale scores were also used in series of CFAs (maximum likelihood estimation based on a Pearson covariance matrix) to examine the goodness-of-fit of statistical models reflecting either the attention-appraisal model, Toronto model, or Amsterdam model. Goodness-of-fit was judged based on the pattern of factor loadings and intercorrelations within each model (Marsh et al., 2004), and three fit indices: the comparative fit index (CFI), the normed fit index (NFI), and the root mean square error of approximation (RMSEA). These three fit indices were chosen as they are considered to be among the best indicators of model fit (Byrne, 2013). CFI and NFI values around $>.90$ were judged to indicate acceptable fit, as were RMSEA values around $<.08$ and less than .10 (Bentler \& Bonnet, 1980; Browne \& Cudeck, 1992; Marsh et al., 2004). Models were also directly compared using the Akaike information criterion (AIC); lower AIC values indicate a better fitting model (Byrne, 2013). Factor loadings $>.40$ were considered meaningful loadings; this cut-off was selected because it has been endorsed by various authors (e.g., Matsunaga, 2010; Stevens, 1992) and is the most commonly used cut-off within psychometric literature (e.g., Bagby et al., 2006; Kooiman et al., 2002). When discussing the observed variables and latent factors within our CFA models, for sake of clarity, observed variables or indicators are written in capital letters and not surrounded by apostrophes (e.g., D-ANALYSING) and latent factors are surrounded by apostrophes (e.g., 'EOT').

All CFA models included five first-order factors: 'DIF', 'DDF', 'EOT', 'DFAN', and 'negative reactivity'. Each latent factor had three observed variables or indicators (subscales). As we administered only two subscales measuring 'DDF' and one subscale measuring 'DFAN', following the recommendations of Gorsuch (1983), to ensure that we had at least 
three indicators for these factors we divided some subscales into parcels ${ }^{9}$. For 'DDF', we created two parcels of items from the BVAQ D-VERBALISING subscale; D-

VERBALISING parcel 1 was composed of the first four items of this subscale, and parcel 2 was composed of the last four items. For 'DFAN', we created three parcels of items from the BVAQ D-FANTASISING subscale; D-FANTASISING parcel 1 was composed of the first three items from this subscale, parcel 2 from the middle three items, and parcel 3 from the last two items.

The five first-order factors in each model were composed of the following subscales as their indicators: 'DIF' (T-DIF, D-IDENTIFYING, and CLARITY), 'DDF' (T-DDF, DVERBALISING parcel 1, and parcel 2), 'EOT’ (T-EOT, D-ANALYSING, and AWARENESS), 'DFAN' (D-FANTASISING parcel 1, parcel 2, and parcel 3), 'negative reactivity’ (NEGATIVE-ACTIVATION, NEGATIVE-INTENSITY, and NEGATIVEDURATION $)^{10}$. All indicators were reasonably normally distributed (maximum skewness $=$ .84 , maximum kurtosis $=-.82$ ). The tested models differed in their higher-order factor structure so as to reflect either the attention-appraisal model, the Toronto model, or the Amsterdam model (see Figure 5.3).

Model 1 was based on the attention-appraisal model. In Model 1, the 'DIF', 'DDF' and 'EOT' factors were specified to load on a higher-order 'alexithymia' factor, whilst the

\footnotetext{
${ }^{9}$ The BVAQ D-VERBALISING subscale was selected for item parcelling because it had the most items of the DDF subscales, and the first and last 20 items of the BVAQ are often administered separately as parallel short versions (Zech et al., 1999). The items of those subscales selected for parcelling have demonstrated unidimensionality in various samples (e.g., Vorst \& Bermond, 2001). In our sample, a single factor solution accounted for $50.96 \%$ of the variance in D-VERBALISING item scores, and $43.36 \%$ of the variance in DFANTASISING item scores.

${ }^{10}$ Although Vorst and Bermond (2001) discuss DEMO as being a unidimensional construct in their theoretical model, more recent work has shown negative reactivity and positive reactivity to be separable dimensions (Becerra et al., 2017). For this reason, only negative reactivity was included within our CFA models. We chose to include negative reactivity rather than positive reactivity, because most of the BVAQ D-EMOTIONALISING items refer to negative emotions, hence we considered negative reactivity to be closer to how Vorst and Bermond (2001) conceptualised DEMO within their model. The BVAQ D-EMOTIONALISING subscale was not included as an indicator of negative reactivity in any of our CFA models, because of our theoretical concerns about this subscale's construct validity and its statistical performance in our EFA.
} 
'DFAN' and 'negative reactivity' factors were allowed to correlate with this higher-order factor and with each other. Model 2a and Model $2 \mathrm{~b}$ were based on the Toronto model. In Model 2a, the 'DIF', 'DDF', 'EOT' and 'DFAN' factors were specified to load on a higherorder 'alexithymia' factor, and the 'negative reactivity' factor was allowed to correlate with this higher-order factor. In Model 2b, the 'DIF' and 'DDF' factors were specified to load on a higher-order 'affect awareness' factor and the 'EOT' and 'DFAN' factors were specified to load on a higher-order 'operative thinking' factor, these higher-order factors were allowed to correlate with each other and the 'negative reactivity' factor. Model 3 was based on the Amsterdam model. In Model 3, the 'DIF', 'DDF' and 'EOT' factors were specified to load on a higher-order 'cognitive alexithymia' factor, and the 'DFAN' and 'negative reactivity' factors were specified to load on a higher-order 'affective alexithymia' factor, these higherorder factors were allowed to correlate. As a comparative baseline, we also tested the fit of a five-factor correlated model with no higher-order structure imposed (Model 4). 

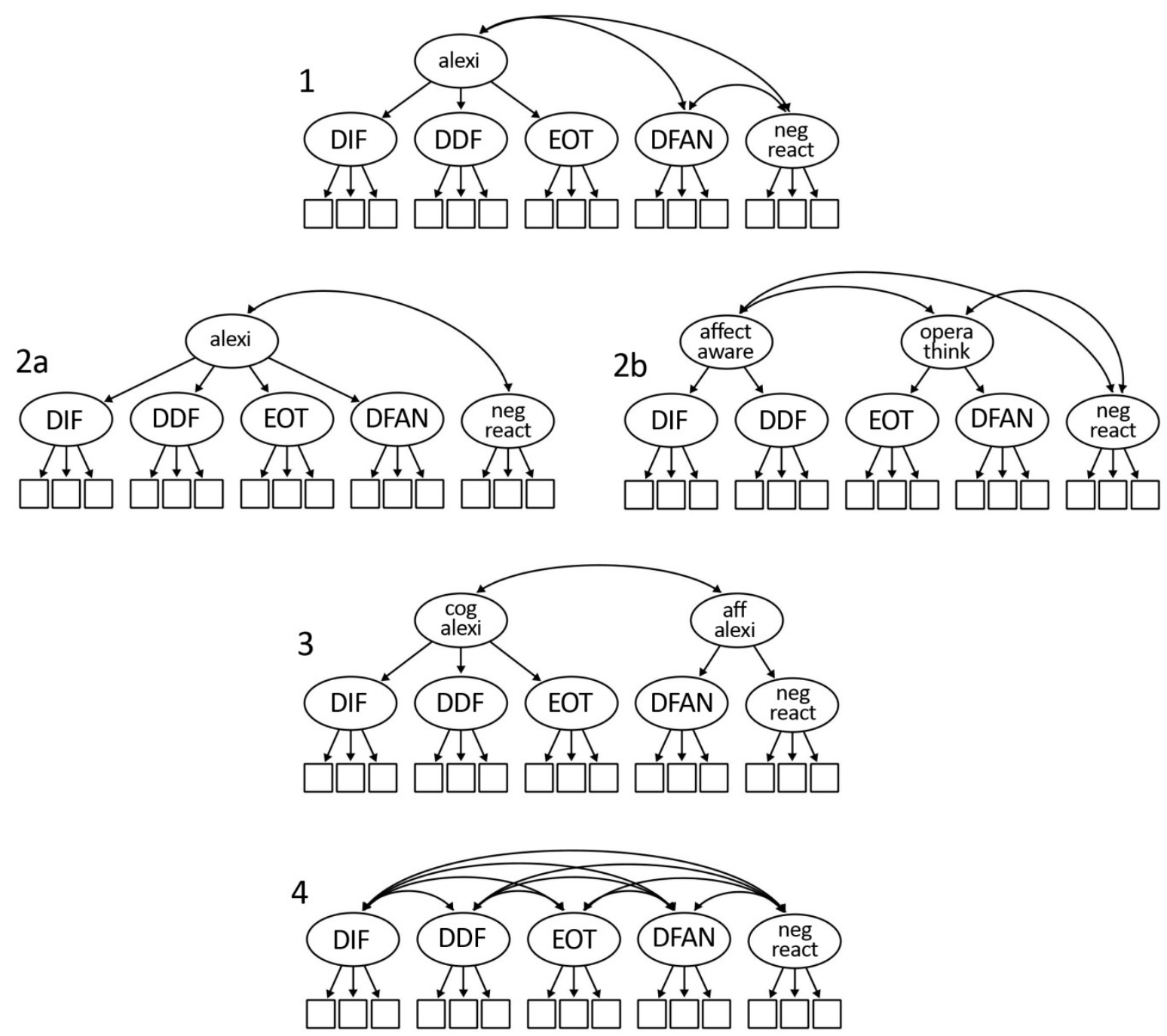

Figure 5.3. A visual representation of the tested CFA models, designed to represent either the attention-appraisal model (Model 1), Toronto model (Model 2a and Model 2b), Amsterdam model (Model 3), or a correlated model used as a comparative baseline (Model 4). Squares indicate observed variables/indicators, ellipses indicate latent variables. Each observed variable had an error term (not displayed). DIF $=$ difficulty identifying feelings, $\mathrm{DDF}=$ difficulty describing feelings, $\mathrm{EOT}=$ externally orientated thinking, DFAN = difficulty fantasising, neg react $=$ negative reactivity, alexi $=$ alexithymia, affect aware $=$ affect awareness, opera think $=$ operative thinking, $\operatorname{cog}$ alexi $=$ cognitive alexithymia, aff alexi = affective alexithymia. Latent variables were comprised of the following subscales (from the TAS-20, BVAQ, DERS, and PERS) as their observed variables: 'DIF' $=$ T-DIF, D-IDENTIFYING, CLARITY; 'DDF' = T-DDF, D-VERBALISING parcel 1, and parcel 2; 'EOT' = T-EOT, D-ANALYSING, AWARENESS; 'DFAN' = D-FANTASISING parcel 1, parcel 2, and parcel 3; 'negative reactivity' = NEGATIVE-ACTIVATION, NEGATIVE-INTENSITY, NEGATIVE-DURATION. 


\section{Results}

\section{Pearson correlations and exploratory factor analysis}

Descriptive statistics for the sample are presented in Table 5.1. All DIF, DDF and EOT subscales were significantly positively correlated ( $r \mathrm{~s}=.30$ to .63$)$. The BVAQ DFANTASISING subscale, however, was uncorrelated or weakly negatively correlated with the DIF subscales ( $r \mathrm{~s}=-.18$ to -.02$)$, uncorrelated with the DDF subscales ( $r \mathrm{~s}=-.13$ to -.03$)$, and uncorrelated or weakly positively correlated with the EOT subscales ( $r \mathrm{~s}=.09$ to .21$)$. The BVAQ D-FANTASISING subscale was also uncorrelated with all cognitive alexithymia total scale scores (TAS-20 TOTAL SCALE, DERS ALEXITHYMIA COMPOSITE, BVAQ COGNITIVE ALEXITHYMIA; $r \mathrm{~s}=-.06$ to .08$)$. When emotional reactivity was measured via the PERS, all cognitive alexithymia total scale scores were significantly associated with higher levels of negative reactivity $(r \mathrm{~s}=.27$ to .43$)$ and lower levels of positive reactivity ( $r \mathrm{~s}$ $=-.29$ to -.35$)$. Conversely, the BVAQ D-EMOTIONALISING subscale was uncorrelated with most cognitive alexithymia total scale scores $(r \mathrm{~s}=.00$ to .23$)$. A Table containing all Pearson correlations is provided in Appendix C.

Our EFA resulted in a four factor solution (eigenvalues $>1$ ), accounting for $71.97 \%$ of the variance (see Table 5.2). Factor 1, which we name 'difficulty appraising feelings', was comprised of all the DIF and DDF subscales. Factor 2, which we name 'negative reactivity', was comprised of all the PERS negative reactivity subscales. Factor 3, which we name 'positive reactivity', was comprised of all the PERS positive reactivity subscales. Factor 4, which we name 'difficulty attending to feelings', was comprised of all the EOT subscales.

The BVAQ D-FANTASISING and D-EMOTIONALISING subscales did not load substantially on any of the extracted factors. These two subscales loaded mostly on the 'difficulty attending to feelings' factor, but the size of their loading $(<.40)$ was not large enough for it to be considered that this factor accounted for a meaningful amount of variance 
in these subscale scores (Stevens, 1992). Of import, the BVAQ D-EMOTIONALISING

subscale did not load substantially on either of the PERS emotional reactivity factors, suggesting that the BVAQ D-EMOTIONALISING subscale was not measuring the same construct as the PERS. Thus, in our EFA, DFAN and DEMO were not part of the same latent structure as DIF, DDF or EOT, nor was DFAN part of the same latent structure as negative or positive reactivity.

Table 5.1

Descriptive Statistics and Cronbach's Alpha ( $\alpha$ ) Internal Reliability

Coefficients for the Administered Measures

\begin{tabular}{|c|c|c|c|}
\hline Scale/subscale & $M$ & $S D$ & $\alpha$ \\
\hline \multicolumn{4}{|l|}{ TAS-20 } \\
\hline Total scale & 48.12 & 12.29 & .87 \\
\hline T-DIF & 14.98 & 6.11 & .87 \\
\hline $\mathrm{T}-\mathrm{DDF}$ & 13.00 & 4.80 & .83 \\
\hline T-EOT & 20.14 & 4.47 & .59 \\
\hline \multicolumn{4}{|l|}{ BVAQ } \\
\hline Cognitive alexithymia & 61.18 & 14.20 & .88 \\
\hline Affective alexithymia & 42.46 & 9.00 & .76 \\
\hline D-Identifying & 18.66 & 5.19 & .75 \\
\hline D-Verbalising & 23.57 & 7.12 & .86 \\
\hline D-Analysing & 18.94 & 5.39 & .77 \\
\hline D-Fantasising & 21.17 & 6.34 & .79 \\
\hline D-Emotionalising & 21.29 & 5.31 & .71 \\
\hline \multicolumn{4}{|l|}{ DERS } \\
\hline Alexithymia composite & 25.77 & 7.59 & .86 \\
\hline Awareness & 15.71 & 5.34 & .84 \\
\hline Clarity & 10.07 & 3.69 & .82 \\
\hline \multicolumn{4}{|l|}{ PERS } \\
\hline General positive reactivity & 51.98 & 8.93 & .92 \\
\hline Positive-activation & 17.41 & 3.65 & .77 \\
\hline Positive-intensity & 15.65 & 3.03 & .84 \\
\hline Positive-duration & 18.92 & 3.64 & .83 \\
\hline General negative reactivity & 43.98 & 13.23 & .94 \\
\hline Negative-activation & 14.37 & 4.81 & .86 \\
\hline Negative-intensity & 15.47 & 4.58 & .86 \\
\hline Negative-duration & 14.13 & 4.93 & .88 \\
\hline
\end{tabular}

Note. TAS-20 $=$ Toronto Alexithymia Scale-20, BVAQ = Bermond-Vorst Alexithymia Questionnaire, DERS $=$ Difficulties in Emotion Regulation Scale, PERS $=$ Perth Emotional Reactivity Scale. 
Table 5.2

Factor loadings from an exploratory factor analysis of the subscales of the TAS-20, BVAQ, DERS and PERS

\begin{tabular}{lcccc}
\hline Subscale & $\begin{array}{c}\text { Factor 1 } \\
\text { 'difficulty } \\
\text { appraising } \\
\text { feelings' }\end{array}$ & $\begin{array}{c}\text { Factor } 2 \\
\text { 'negative } \\
\text { reactivity' }\end{array}$ & $\begin{array}{c}\text { Factor } 3 \\
\text { 'positive } \\
\text { reactivity' }\end{array}$ & $\begin{array}{c}\text { Factor } 4 \\
\text { 'difficulty } \\
\text { attending to } \\
\text { feelings' }\end{array}$ \\
\hline BVAQ & & & & \\
D-Identifying & $\mathbf{. 6 1 6}$ & .171 & -.034 & .148 \\
D-Verbalising & $\mathbf{. 6 8 6}$ & -.113 & -.109 & .114 \\
D-Analysing & .209 & .166 & .032 & $\mathbf{. 8 6 6}$ \\
D-Fantasising & -.199 & -.103 & -.033 & .362 \\
D-Emotionalising & -.076 & -.232 & -.305 & .372 \\
TAS-20 & & & & \\
T-DIF & $\mathbf{. 6 4 0}$ & .270 & .030 & -.071 \\
T-DDF & $\mathbf{. 9 3 0}$ & -.139 & -.031 & -.044 \\
T-EOT & .336 & .083 & .060 & $\mathbf{. 5 7 2}$ \\
DERS & & & & \\
Clarity & $\mathbf{. 7 4 9}$ & .130 & -.016 & .009 \\
Awareness &. $\mathbf{4 1 7}$ & -.013 & -.078 & $\mathbf{. 5 2 2}$ \\
PERS & & & & \\
Positive-activation & -.059 & -.141 & $\mathbf{. 8 8 2}$ & .073 \\
Positive-intensity & .011 & .136 & $\mathbf{. 7 2 1}$ & -.005 \\
Positive-duration & -.118 & -.352 & $\mathbf{. 7 2 0}$ & -.003 \\
Negative-activation & .007 & $\mathbf{. 8 7 4}$ & -.029 & .080 \\
Negative-intensity & -.060 & $\mathbf{. 8 9 5}$ & -.025 & -.075 \\
Negative-duration & .033 & $\mathbf{. 8 7 2}$ & -.070 & .020 \\
\hline
\end{tabular}

Note. Factor loadings $\geq .40$ are in boldface. Intercorrelations between the extracted factors were as follows; $\mathrm{F} 1$ and $\mathrm{F} 2=.46 ; \mathrm{F} 1$ and $\mathrm{F} 3=-.20 ; \mathrm{F} 1$ and $\mathrm{F} 4=.27 ; \mathrm{F} 2$ and $\mathrm{F} 3=-.05 ; \mathrm{F} 2$ and F4 $=-.19 ; \mathrm{F} 3$ and F4 $=-.29$. TAS-20 $=$ Toronto Alexithymia Scale-20, BVAQ $=$ Bermond-Vorst Alexithymia Questionnaire, DERS = Difficulties in Emotion Regulation Scale, PERS $=$ Perth Emotional Reactivity Scale.

\section{Confirmatory factor analyses}

Of the theoretically informed CFA models, Model 1 based on the attention-appraisal model appeared to be the best fit to the data (for fit index values, factor loadings, and estimated factor intercorrelations for each model, see Tables 5.3, 5.4 and 5.5, respectively). In Model 1, the latent 'DIF', 'DDF' and 'EOT' factors all loaded strongly (factor loadings = $.727-.853)$ on the higher-order 'alexithymia' factor, indicating that these three first-order 
factors could be components of a common higher-order construct. Models based on the Toronto model exhibited poorer levels of fit. The main source of misspecification appeared to be the latent 'DFAN' factor. In Model 2a, the 'DFAN' factor loaded poorly and nonsignificantly (factor loading = -.102) on the higher-order 'alexithymia' factor, and in Model $2 \mathrm{~b}$, the 'EOT' factor loaded poorly (factor loading $=.256$ ) with the 'DFAN' factor on the higher-order 'operative thinking' factor. Thus, the 'DFAN' factor did not appear to be part of the same latent structure as 'DIF', 'DDF' or 'EOT'. Model 3, based on the Amsterdam model, similarly exhibited poorer levels of fit. The higher-order 'affective alexithymia' factor (representing difficulties fantasising and low negative reactivity) in this model was strongly negatively correlated with the higher-order 'cognitive alexithymia' factor (estimated $r=$ .527); thus, these two higher-order factors were not orthogonal or positively correlated. Indeed, an inspection of the factor intercorrelations within the correlated baseline model (Model 4) revealed that the 'negative reactivity' factor was significantly positively associated with 'DIF', 'DDF' and 'EOT', and generally correlated more so with these cognitive alexithymia components than it did with 'DFAN'. In other words, across our CFAs, cognitive alexithymia was associated with higher levels of negative reactivity, not less. 
Table 5.3

Goodness-of-Fit Index Values for the Examined CFA Models

\begin{tabular}{|c|c|c|c|c|c|c|}
\hline Model & $\chi^{2}(d f)$ & $p$ & CFI & NFI & RMSEA $(90 \% \mathrm{CI})$ & AIC \\
\hline \multicolumn{7}{|l|}{ Attention-appraisal model } \\
\hline Model 1 & $445.196(85)$ & .000 & .896 & .875 & $.107(.098-.117)$ & 515.196 \\
\hline Model 1 (correlated error) & $307.819(79)$ & .000 & .934 & .914 & $.089(.079-.099)$ & 389.819 \\
\hline \multicolumn{7}{|l|}{ Toronto model } \\
\hline Model 2a & $458.382(85)$ & .000 & .892 & .872 & $.109(.100-.119)$ & 528.382 \\
\hline Model 2a (correlated error) & $330.777(79)$ & .000 & .927 & .907 & $.093(.083-.104)$ & 412.777 \\
\hline Model 2b & $579.531(85)$ & .000 & .857 & .838 & $.126(.116-.136)$ & 649.531 \\
\hline Model 2b (correlated error) & $448.075(79)$ & .000 & .894 & .875 & $.113(.098-123)$ & 530.075 \\
\hline \multicolumn{7}{|l|}{ Amsterdam model } \\
\hline Model 3 & $476.085(86)$ & .000 & .887 & .867 & $.111(.102-.121)$ & 544.085 \\
\hline Model 3 (correlated error) & $310.652(80)$ & .000 & .933 & .913 & $.089(.078-.099)$ & 390.652 \\
\hline \multicolumn{7}{|l|}{ Baseline correlated model } \\
\hline Model 4 & $357.153(80)$ & .000 & .920 & .900 & $.097(.087-.108)$ & 437.153 \\
\hline Model 4 (correlated error) & $227.055(74)$ & .000 & .956 & .936 & $.075(.064-.086)$ & 319.055 \\
\hline
\end{tabular}

Note. Models labelled with 'correlated error' included minor model modifications whereby some indicator error terms were allowed to correlate. These modifications were added because modification indices indicated that, across all the examined models, some error terms were substantially correlated; these error terms were between those indicators/subscales that came from the same measure (e.g., the TAS-20) and thus appeared to represent some common method variance. On these grounds, we considered these modifications to be theoretically justifiable (Podsakoff et al., 2003). The error terms of the following indicators were allowed to correlate: T-DIF and T-DDF; D-VERBALISING parcel 1 and D-VERBALISING parcel 2; D-VERBALISING parcel 2 and D-ANALYSING; D-ANALYSING and DFANTASISING parcel 1; D-ANALYSING and D-FANTASISING parcel 3; D-FANTASISING parcel 2 and D-FANTASISING parcel 3. CFI = comparative fit index, NFI = normed fit index, RMSEA = root mean square error of approximation, $\mathrm{AIC}=$ Akaike information criterion, $\mathrm{CI}=$ confidence interval. 
Table 5.4

Standardised Factor Loadings for the Indicators and Latent Factors in CFA Models Based on the Attention-Appraisal Model, Toronto Model, and Amsterdam Model

\begin{tabular}{|c|c|c|c|c|}
\hline \multirow[b]{2}{*}{$\begin{array}{l}\text { Observed variables and latent } \\
\text { factors }\end{array}$} & \multirow{2}{*}{$\begin{array}{c}\begin{array}{c}\text { Attention- } \\
\text { appraisal } \\
\text { model }\end{array} \\
\text { Model } 1\end{array}$} & \multicolumn{2}{|c|}{ Toronto model } & \multirow{2}{*}{$\begin{array}{c}\begin{array}{c}\text { Amsterdam } \\
\text { model }\end{array} \\
\text { Model } 3\end{array}$} \\
\hline & & Model 2a & Model 2b & \\
\hline & Alexithymia $^{a}$ & Alexithymia $^{\mathrm{a}}$ & $\begin{array}{c}\text { Affect } \\
\text { awareness }^{\mathrm{a}}\end{array}$ & $\begin{array}{l}\text { Cognitive } \\
\text { alexithymia }\end{array}$ \\
\hline 'DIF' factor & $.853^{\mathrm{b}}$ & $.953^{\mathrm{b}}$ & $.919^{\mathrm{b}}$ & $.861^{\mathrm{b}}$ \\
\hline T-DIF & .767 & .774 & .788 & .771 \\
\hline D-Identifying & .815 & .823 & .777 & .816 \\
\hline \multirow[t]{2}{*}{ Clarity } & .862 & .862 & .860 & .867 \\
\hline & Alexithymia $^{a}$ & Alexithymia $^{a}$ & $\begin{array}{c}\text { Affect } \\
\text { awareness }^{\mathrm{a}}\end{array}$ & $\begin{array}{l}\text { Cognitive } \\
\text { alexithymia }\end{array}$ \\
\hline 'DDF' factor & $.823^{\mathrm{b}}$ & $.734^{\mathrm{b}}$ & $.774^{\mathrm{b}}$ & $.831^{\mathrm{b}}$ \\
\hline T-DDF & .876 & .871 & .899 & .881 \\
\hline D-Verbalising parcel 1 & .855 & .855 & .855 & .855 \\
\hline \multirow[t]{2}{*}{ D-Verbalising parcel 2} & .856 & .850 & .847 & .854 \\
\hline & Alexithymia ${ }^{a}$ & Alexithymia $^{a}$ & $\begin{array}{l}\text { Operative } \\
\text { thinking }\end{array}$ & $\begin{array}{l}\text { Cognitive } \\
\text { alexithymia }\end{array}$ \\
\hline 'EOT' factor & $.727^{\mathrm{b}}$ & $.659^{\mathrm{b}}$ & $.256^{\mathrm{b}}$ & $.720^{\mathrm{b}}$ \\
\hline T-EOT & .751 & .750 & .741 & .752 \\
\hline D-Analysing & .846 & .847 & .877 & .846 \\
\hline \multirow[t]{2}{*}{ Awareness } & .808 & .809 & .783 & .812 \\
\hline & - & Alexithymia $^{a}$ & $\begin{array}{l}\text { Operative } \\
\text { thinking }\end{array}$ & $\begin{array}{l}\text { Affective } \\
\text { alexithymia }^{\mathrm{a}}\end{array}$ \\
\hline 'DFAN' factor & - & $-.102 * b$ & $.860^{\mathrm{b}}$ & $.545^{\mathrm{b}}$ \\
\hline D-Fantasising parcel 1 & .594 & .587 & .596 & .708 \\
\hline D-Fantasising parcel 2 & .857 & .846 & .852 & .848 \\
\hline \multirow[t]{2}{*}{ D-Fantasising parcel 3} & .778 & .793 & .782 & .802 \\
\hline & - & - & - & $\begin{array}{l}\text { Affective } \\
\text { alexithymia }\end{array}$ \\
\hline 'Negative reactivity' factor & - & - & - & $-.802^{\mathrm{b}}$ \\
\hline Negative-activation & .870 & .874 & .870 & .875 \\
\hline Negative-intensity & .885 & .881 & .884 & .895 \\
\hline Negative-duration & .892 & .892 & .883 & .896 \\
\hline
\end{tabular}

Note. ${ }^{*} p>.05$. ${ }^{\mathrm{a}}$ Label of higher-order factor. ${ }^{\mathrm{b}}$ Factor loading of first-order factor on higher-order factor. Factor loadings $<.40$ are in boldface. 
Table 5.5

Estimated Factor Intercorrelations for CFA Models Based on the Attention-Appraisal

Model, Toronto Model, and Amsterdam Model

\begin{tabular}{|c|c|c|c|c|}
\hline \multirow[b]{2}{*}{ Model/factor } & \multicolumn{4}{|c|}{ Factor } \\
\hline & F1 & $\mathrm{F} 2$ & F3 & $\mathrm{F} 4$ \\
\hline \multicolumn{5}{|l|}{ Attention-appraisal model } \\
\hline \multicolumn{5}{|l|}{ Model 1} \\
\hline F1 'alexithymia' & - & - & - & - \\
\hline F2 'DFAN' & -.029 & - & - & - \\
\hline F3 'negative reactivity' & $.449 * * *$ & $-.251 * * *$ & - & - \\
\hline \multicolumn{5}{|l|}{ Toronto model } \\
\hline \multicolumn{5}{|l|}{ Model 2a } \\
\hline F1 'alexithymia' & - & - & - & - \\
\hline F2 'negative reactivity' & $.513 * * *$ & - & - & - \\
\hline \multicolumn{5}{|l|}{ Model 2b } \\
\hline F1 'affect awareness' & - & - & - & - \\
\hline F2 'operative thinking' & -.052 & - & - & - \\
\hline F3 'negative reactivity' & $.551 * * *$ & $-.261 * * *$ & - & - \\
\hline \multicolumn{5}{|l|}{ Amsterdam model } \\
\hline \multicolumn{5}{|l|}{ Model 3} \\
\hline F1 'cognitive alexithymia' & - & - & - & - \\
\hline F2 'affective alexithymia' & $-.527 * * *$ & - & - & - \\
\hline \multicolumn{5}{|l|}{ Baseline correlated model } \\
\hline \multicolumn{5}{|l|}{ Model 4} \\
\hline F1 'DIF' & - & - & - & - \\
\hline $\mathrm{F} 2$ 'DDF' & $.667 * * *$ & - & - & - \\
\hline F3 'EOT' & $.609 * * *$ & $.648 * * *$ & - & - \\
\hline F4 'DFAN' & $-.146^{*}$ & -.045 & $.216^{* *}$ & - \\
\hline F5 'negative reactivity' & $.558 * * *$ & $.290 *$ & $.141^{*}$ & $.253^{* * *}$ \\
\hline
\end{tabular}

Note. $p<.001 * * *, p<.01 * *, p<.05^{*}$.

\section{Discussion}

One purpose of this study was to explore the latent structure of alexithymia. In our sample, we found that DIF, DDF and EOT formed a coherent latent structure, DFAN and 
DEMO were not part of this same latent structure, and alexithymia was associated with higher (not lower) levels of negative reactivity. Our results were, therefore, consistent with the view that the alexithymia construct is comprised of only three components; DIF, DDF and EOT.

This pattern of findings has now emerged across multiple samples and assessment modalities (e.g., Bausch et al., 2011; Taylor et al., 1985; Vorst \& Bermond, 2001; Watters, Taylor, \& Bagby, 2016; Watters, Taylor, Quilty, \& Bagby, 2016), and as such, we think the current body of evidence is, on balance, sufficient to warrant a refinement to the definition of alexithymia; refined to a construct comprised of only DIF, DDF and EOT. This pattern of findings is consistent with the structure specified by our attention-appraisal model of alexithymia, rather than that of the Toronto or Amsterdam models. Consequently, by aligning alexithymia theory with recent advances in the broader emotion regulation field (Gross, 2015a), we think our model might provide a useful framework for understanding and defining the alexithymia phenomenon in future work. When the alexithymia phenomenon is viewed through the lens of our model, this accounts for a number of unresolved issues within the field.

Firstly, by conceptualising alexithymia as a set of difficulties during the emotion valuation process (Gross, 2015a) and linking these difficulties to emotion schemas (Lane \& Schwartz, 1987), our model can account for why most empirical work finds DFAN to not be part of the alexithymia construct; because unlike DIF, DDF and EOT, the extent to which one engages in daydreaming and fantasy would not seem to be dependent on the developmental level of one's emotion schemas (see also, Stawarczyk, Majerus, Van der Linden, \& D'Argembeau, 2012).

Secondly, by positioning alexithymia within Gross's (2015a) extended process model of emotion regulation, our model can account for why empirical work commonly finds 
alexithymia to be associated with high levels of negative reactivity. Namely, if one accepts that people are generally driven by hedonistic motivations to feel pleasure and avoid pain, then most emotion regulation attempts will be focused on up-regulating positive feelings and down-regulating negative feelings (Gross, 2014). Consequently, people who have poor emotion regulation skills would be expected to experience negative feelings more intensely and positive feelings less intensely than people who are adept at emotion regulation. In turn, because alexithymia constitutes difficulties during the emotion valuation process that is responsible for activating regulatory attempts, alexithymic people would be expected to have poorer emotion regulation skills and less capacity to control their negative feelings. Indeed, alexithymia has been consistently associated with maladaptive emotion regulation attempts (e.g., Swart, Kortekaas, \& Aleman, 2009) and elsewhere has been described as a rate-limiting factor for successful emotion regulation (Gross, 2014). In other words, from this perspective, it is not the absence of affect that characterises alexithymia, but rather, the undifferentiated structure of the affect. This also accounts for why high levels of alexithymia are commonly observed in patients with psychopathologies characterised by emotion dysregulation and negative reactivity (New et al., 2012; Leweke, Leichsenring, Kruse, \& Hermes, 2012).

Thirdly, by making a distinction between ability deficit alexithymia and avoidance alexithymia, our model informs the debate within the literature with respect to whether alexithymia should be considered a deficit (e.g., Taylor et al., 1999) or a defence (e.g., Marchesi, Ossola, Tonna, \& De Panfilis, 2014). Within our model, alexithymia is both, and it is the combination of ability deficits and avoidant defences that determines one's overall level of alexithymia. Similarly, this conceptualisation informs a related debate on whether alexithymia should be considered a stable trait (e.g., Taylor et al., 1999) or a state reaction to distress (e.g., Marchesi et al., 2014). Within our model, one's level of ability deficit alexithymia should be relatively stable, but levels of avoidance alexithymia may increase as a 
regulatory response to unpleasant feelings. This is consistent with the current set of findings from longitudinal studies, which report that alexithymia levels (TAS-20 total scale scores) usually elevate during periods of distress, but nonetheless exhibit an underlying level of relative stability (de Timary, Luts, Hers, \& Luminet, 2008; Luminet et al., 2001; Luminet et al., 2007).

Fourthly, our model could be helpful in informing the treatment of those psychiatric patients who present with high levels of alexithymia. Our model suggests that psychotherapy with such patients should include a focus on developing their emotion schemas and reducing their use of experiential avoidance as an emotion regulation strategy. From this perspective, difficulties attending to and appraising emotions should be targeted by guiding the patient in focusing on the most pertinent features of an emotional response, and guiding them in mentally representing, labelling, and linking the visceral sensations, behavioural tendencies, and eliciting events surrounding this affect. The goal is to facilitate the patient's progression to a higher level of emotional awareness at the attention and appraisal stages of emotion valuation (Gross, 2015a; Lane \& Schwartz, 1987). Psychotherapy techniques compatible with this approach have been described previously by several authors, including Kennedy and Franklin (2002), Lane et al. (2015), Taylor et al. (1999), and Neumann, Malec and Hammond (2017), and we consider mindfulness techniques (e.g., mindfulness of emotions; Harris, 2009) to be of particular relevance. Research on the treatment of alexithymia in the context of psychopathology is still in its relative infancy (Samur et al., 2013), and the attention-appraisal model could provide a useful framework for the design of alexithymia focused psychotherapy programs to be tested in future work.

Fifthly, the TAS-20 (Bagby et al., 1994) has been by far the most widely used measure of alexithymia since its development (Taylor et al., 2016), and because the TAS-20 includes only DIF, DDF and EOT items, most of the alexithymia literature has already 
operationalised the construct in a manner consistent with the three component definition we have suggested in this paper (e.g., Bankier, Aigner, \& Bach, 2001; Leweke et al., 2012; Panayiotou et al., 2015; Parker et al., 2008; Subic-Wrana, Bruder, Thomas, Lane, \& Köhle, 2005; for a review, see Taylor \& Bagby, 2004). The introduction of our model, therefore, reduces the discrepancy that some authors had noted to exist between alexithymia theory and alexithymia measurement (Sifneos, 1996; Bagby, Taylor, Quilty, \& Parker, 2007).

\section{Limitations}

Whilst we consider our paper to make a strong contribution, some limitations of our factor analytic study should be noted. Although we used multiple measures of alexithymia, we used only one measure of DFAN and all our measures were self-report questionnaires. Previous research has shown that the BVAQ DFAN subscale correlates strongly with observer-rated measures of DFAN (e.g., Rosenberg et al., 2016), but nonetheless, it would be ideal for future research of this type to also include observer-rated measures like the TSIA. Our results also only relate to an Australian adult community sample. Similar results to ours have emerged when these measures have been examined in isolation across various populations (e.g., Meganck et al., 2008; Taylor, Bagby, \& Parker, 2003; Bermond et al., 2007), but more research is needed in which multiple measures are administered together. Additionally, whilst our model includes hypotheses about the role of emotion schemas and experiential avoidance in alexithymia, we did not test these mechanisms in this study. As such, from our data, we can only comment on the latent structure of the construct rather than the mechanisms underlying this structure. There is a growing body of work supporting the role of emotion schemas (e.g., Lane et al., 1996; Luminet et al., 2006; Lundh et al., 2002; Suslow \& Junghanns, 2002; Vermeulen et al., 2006) and experiential avoidance (e.g., Bilotta et al., 2015; Coriale et al., 2012; Panayiotou et al., 2015) in alexithymia, but more research of this type is needed to enhance understanding of the theoretical underpinnings of the construct. 
Nonetheless, despite these limitations, in terms of the latent structure of the construct, we think the current body of empirical evidence is sufficient to warrant a refinement to the definition of alexithymia; refined to a construct comprised of DIF, DDF and EOT.

\section{Conclusions}

The latent structure of the alexithymia construct, statistically, appears to be comprised of only DIF, DDF and EOT. We, therefore, recommend that the definition of alexithymia be refined so as to be consistent with the weight of the empirical literature, and include only these three components. This structure is consistent with the specifications of the attentionappraisal model of alexithymia, and by aligning alexithymia theory with recent advances in the broader emotion regulation field (Gross, 2015a), we think that this model provides a useful framework for clinicians and researchers to use and test in future work. 


\section{References}

Abler, B. \& Kessler, H. (2009). Emotion Regulation Questionnaire - Eine deutsche Fassung des ERQ von Gross \& John. Diagnostica, 55, 144-152.

Aldao, A., \& Christensen, K. (2015). Linking the expanded process model of emotion regulation to psychopathology by focusing on behavioral outcomes of regulation Psychological Inquiry, 26, 27-36.

Aldao, A., Gee, D. G., De Los Reyes, A., \& Seager, I. (2016). Emotion regulation as a transdiagnostic factor in the development of internalizing and externalizing psychopathology: Current and future directions. Development and Psychopathology, 28, 927-946.

Aldao, A., \& Nolen-Hoeksema, S. (2010). Specificity of cognitive emotion regulation strategies: A transdiagnostic examination. Behaviour Research and Therapy, 48, 974983.

Aldao, A., Nolen-Hoeksema, S., \& Schweizer, S. (2010). Emotion-regulation strategies across psychopathology: A meta-analytic review. Clinical Psychology Review, 30, 217-237.

Aldao, A., Sheppes, G., \& Gross, J. J. (2015). Emotion regulation flexibility. Cognitive Therapy and Research, 39, 263-278.

American Psychiatric Association (2013). Diagnostic and statistical manual of mental disorders (5th ed.). Washington, DC: Author.

Amodio, D. M., Master, S. L., Yee, C. M., \& Taylor, S. E. (2008). Neurocognitive components of the behavioral inhibition and activation systems: Implications for theories of self-regulation. Psychophysiology, 45, 11-19. 
Australian Bureau of Statistics. (2016). Education and work, Australia (no. 6227.0). Retrieved from http://www.abs.gov.au/ausstats/abs@.nsf/mf/6227.0.

Australian Bureau of Statistics. (2017a). 2016 Census Data Summary: Education qualifications in Australia. Retrieved from http://www.abs.gov.au/AUSSTATS/abs@.nsf/DetailsPage/2071.02016?OpenDocume $\mathrm{nt}$

Australian Bureau of Statistics. (2017b). 2016 Census data summary: Cultural diversity in Australia. Retrieved from http://www.abs.gov.au/AUSSTATS/abs@.nsf/DetailsPage/2071.02016?OpenDocume nt

Bach, M., Bach, D., De Zwaan, M., Serim, M., \& Böhmer, F. (1996). Validation of the German version of the 20-item Toronto alexithymia scale in normal persons and psychiatric patients. Psychotherapie, Psychosomatik, Medizinische Psychologie, 46, $23-28$.

Bachorowski, J. A., \& Braaten, E. B. (1994). Emotional intensity: Measurement and theoretical implications. Personality and Individual Differences, 17, 191-199.

Badcock, J. C., Paulik, G., \& Maybery, M. T. (2011). The role of emotion regulation in auditory hallucinations. Psychiatry Research, 185, 303-308.

Bagby, R. M., Parker, J. D., \& Taylor, G. J. (1994). The twenty-item Toronto alexithymia scale-I. Item selection and cross-validation of the factor structure. Journal of Psychosomatic Research, 38, 23-32.

Bagby, R. M., Quilty, L. C., Taylor, G. J., Grabe, H. J., Luminet, O., Verissimo, R., ... Vanheule, S. (2009). Are there subtypes of alexithymia? Personality and Individual Differences, 47, 413-418. 
Bagby, R. M., Taylor, G. J., Parker, J. D., \& Dickens, S. E. (2006). The development of the Toronto structured interview for alexithymia: Item selection, factor structure, reliability and concurrent validity. Psychotherapy and Psychosomatics, 75, 25-39.

Bagby, R. M., Taylor, G. J., Quilty, L. C., \& Parker, J. D. (2007). Reexamining the factor structure of the 20-item Toronto alexithymia scale: Commentary on Gignac, Palmer, and Stough. Journal of Personality Assessment, 89, 258-264.

Bailey, P. E., \& Henry, J. D. (2007). Alexithymia, somatization and negative affect in a community sample. Psychiatry Research, 150, 13-20.

Balzarotti, S., Gross, J. J., \& John, O. P. (2010). An Italian adaptation of the Emotion Regulation Questionnaire. European Journal of Psychological Assessment, 26, 6167.

Bankier, B., Aigner, M., \& Bach, M. (2001). Alexithymia in DSM-IV disorder: Comparative evaluation of somatoform disorder, panic disorder, obsessive-compulsive disorder, and depression. Psychosomatics, 42, 235-240.

Bardeen, J. R., Fergus, T. A., \& Orcutt, H. K. (2012). An examination of the latent structure of the difficulties in emotion regulation scale. Journal of Psychopathology and Behavioral Assessment, 34, 382-392.

Bardeen, J. R., Fergus, T. A., Hannan, S. M., \& Orcutt, H. K. (2016). Addressing psychometric limitations of the difficulties in emotion regulation scale through item modification. Journal of Personality Assessment, 98, 298-309.

Barlow, D. H., Farchione, T. J., Bullis, J. R., Gallagher, M. W., Murray-Latin, H., SauerZavala, S., ... \& Ametaj, A. (2017). The unified protocol for transdiagnostic treatment of emotional disorders compared with diagnosis-specific protocols for anxiety disorders: A randomized clinical trial. JAMA Psychiatry, 74, 875-884. 
Barlow, D. H., Farchione, T. J., Fairholme, C. P., Ellard, K. K., Boisseau, C. L., Allen, L. B., \& May, J. T. E. (2010). Unified protocol for transdiagnostic treatment of emotional disorders: Therapist guide. New York, NY: Oxford University Press.

Barrett, L. F. (2009). The future of psychology: Connecting mind to brain. Perspectives in Psychological Science, 4, 326-339.

Barrett, L. F., Gross, J., Christensen, T. C., \& Benvenuto, M. (2001). Knowing what you're feeling and knowing what to do about it: Mapping the relation between emotion differentiation and emotion regulation. Cognition and Emotion, 15, 713-724.

Bausch, S., Stingl, M., Hartmann, L. C., Leibing, E., Leichsenring, F., Kruse, J., ... Leweke, F. (2011). Alexithymia and script driven emotional imagery in healthy female subjects: No support for deficiencies in imagination. Scandinavian Journal of Psychology, 52, 179-184.

Becerra, R., \& Campitelli, G. (2013). Emotional reactivity: Critical analysis and proposal of a new scale. International Journal of Applied Psychology, 3, 161-168.

Becerra, R., Amos, A., \& Jongenelis, S. (2002). Organic alexithymia: A study of acquired emotional blindness. Brain Injury, 16, 633-645.

Becerra, R., Cruise, K., Murray, G., Bassett, D., Harms, C., Allan, A., \& Hood, S. (2013). Emotion regulation in bipolar disorder: Are emotion regulation abilities less compromised in euthymic bipolar disorder than unipolar depressive or anxiety disorders? Open Journal of Psychiatry, 3, 1-7.

Becerra, R., Preece, D., Campitelli, G., \& Scott-Pillow, G. (2017). The assessment of emotional reactivity across negative and positive emotions: Development and validation of the Perth Emotional Reactivity Scale (PERS). Assessment, 1-13. doi: $10.1177 / 1073191117694455$ 
Beck, A. T., \& Dozois, D. J. (2011). Cognitive therapy: Current status and future directions. Annual Review of Medicine, 62, 397-409.

Bentler, P. M., \& Bonett, D. G. (1980). Significance tests and goodness of fit in the analysis of covariance structures. Psychological Bulletin, 88, 588-606.

Berenbaum, H. (1996). Childhood abuse, alexithymia and personality disorder. Journal of Psychosomatic Research, 41, 585-595.

Bermond, B., Clayton, K., Liberova, A., Luminet, O., Maruszewski, T., Ricci Bitti, P. E., \& Wicherts, J. (2007). A cognitive and an affective dimension of alexithymia in six languages and seven populations. Cognition and Emotion, 21, 1125-1136.

Bermond, B., Oosterveld, P., \& Vorst, H. C. M. (2015). Measures of alexithymia. In G. J. Boyle, D. H. Saklofske, \& G. Matthews (Eds.), Measures of personality and social psychological constructs (pp. 227-256). San Diego: Academic.

Bermond, B., Vorst, H. C., Vingerhoets, A. J., \& Gerritsen, W. (1999). The Amsterdam alexithymia scale: Its psychometric values and correlations with other personality traits. Psychotherapy and Psychosomatics, 68, 241-251.

Bilotta, E., Giacomantonio, M., Leone, L., Mancini, F., \& Coriale, G. (2015). Being alexithymic: Necessity or convenience. Negative emotionality $\times$ avoidant coping interactions and alexithymia. Psychology and Psychotherapy: Theory, Research and Practice, 89, 261-275.

Bonanno, G. A., \& Burton, C. L. (2013). Regulatory flexibility: An individual differences perspective on coping and emotion regulation. Perspectives on Psychological Science, $8,591-612$.

Boss, A. D., \& Sims, H. P. (2008). Everyone fails! Using emotion regulation and selfleadership for recovery. Journal of Managerial Psychology, 23, 135-150. 
Boyes, M. E., Carmody, T. M., Clarke, P. J., \& Hasking, P. A. (2017). Emotional reactivity and perseveration: Independent dimensions of trait positive and negative affectivity and differential associations with psychological distress. Personality and Individual Differences, 105, 70-77.

Brackett, M. A., Rivers, S. E., \& Salovey, P. (2011). Emotional intelligence: Implications for personal, social, academic, and workplace success. Social and Personality Psychology Compass, 5, 88-103.

Bradley, M. M., \& Lang, P. J. (2007). The international affective picture system (IAPS) in the study of emotion and attention. In J. A. Coan, \& J. J. B. Allen (Eds.). Handbook of emotion elicitation and assessment (pp. 29-46). New York, NY: Oxford University Press.

Bressi, C., Taylor, G., Parker, J., Bressi, S., Brambilla, V., Aguglia, E., et al. (1996). Cross validation of the factor structure of the 20-item Toronto alexithymia scale: An Italian multicenter study. Journal of Psychosomatic Research, 41, 551-559.

Brown, T. A. (2007). Temporal course and structural relationships among dimensions of temperament and DSM-IV anxiety and mood disorder constructs. Journal of Abnormal Psychology, 116, 313-328.

Brown, T. A. (2014). Confirmatory factor analysis for applied research. New York: Guilford Publications.

Brown, T. A., Chorpita, B. F., \& Barlow, D. H. (1998). Structural relationships among dimensions of the DSM-IV anxiety and mood disorders and dimensions of negative affect, positive affect, and autonomic arousal. Journal of Abnormal Psychology, 107, 179-192.

Browne, M. W., \& Cudeck, R. (1992). Alternate ways of assessing model fit. Sociological Methods \& Research, 21, 230-258. 
Bucci, W. (1997). Symptoms and symbols: A multiple code theory of somatization. Psychoanalytic Inquiry, 17, 151-172.

Bullis, J. R., Sauer-Zavala, S., Bentley, K. H., Thompson-Hollands, J., Carl, J. R., \& Barlow, D. H. (2015). The unified protocol for transdiagnostic treatment of emotional disorders: preliminary exploration of effectiveness for group delivery. Behavior Modification, 39, 295-321.

Butler, E. A., Lee, T. L., \& Gross, J. J. (2007). Emotion regulation and culture: Are the social consequences of emotion suppression culture-specific? Emotion, 7, 30-48.

Bydlowski, S., Corcos, M., Jeammet, P., Paterniti, S., Berthoz, S., Laurier, C., et al. (2005). Emotional-processing deficits in eating disorders. International Journal of Eating Disorders, 37, 321-329.

Byrne, B. M. (2013). Structural equation modeling with LISREL, PRELIS, and SIMPLIS: Basic concepts, applications, and programming. New York, NY: Psychology Press.

Byrne, B. M. (2016). Structural equation modeling with AMOS: Basic concepts, applications, and programming. New York, NY: Routledge.

Cabello, R., Salguero, J. M., Fernández-Berrocal, P., \& Gross, J. J. (2013). A Spanish adaptation of the emotion regulation questionnaire. European Journal of Psychological Assessment, 29, 234-240.

Campbell-Sills, L., \& Barlow, D. H. (2007). Incorporating emotion regulation into conceptualizations and treatments of anxiety and mood disorders. In J. J. Gross (Ed.). Handbook of emotion regulation (pp. 542-559). New York: Guilford Press.

Campbell-Sills, L., Ellard, K. K., \& Barlow, D. H. (2014). Emotion regulation in anxiety disorders. In J. J. Gross (Ed.), Handbook of emotion regulation (pp. 393-412). New York, NY: Guilford Press. 
Campos, A., Chiva, M., \& Moreau, M. (2000). Alexithymia and mental imagery. Personality and Individual Differences, 29, 787-791.

Capaldi, D. M., \& Rothbart, M. K. (1992). Development and validation of an early adolescent temperament measure. The Journal of Early Adolescence, 12, 153-173.

Caretti, V., Porcelli, P., Solano, L., Schimmenti, A., Bagby, R. M., \& Taylor, G. J. (2011). Reliability and validity of the Toronto structured interview for alexithymia in a mixed clinical and nonclinical sample from Italy. Psychiatry Research, 187, 432-436.

Carver, C. S., Scheier, M. F., \& Weintraub, J. K. (1989). Assessing coping strategies: A theoretically based approach. Journal of Personality and Social Psychology, 56, 267283.

Catanzaro, S. J., \& Mearns, J. (1990). Measuring generalized expectancies for negative mood regulation: Initial scale development and implications. Journal of Personality Assessment, 54, 546-563.

Cattell, R. B. (1943). The description of personality: Basic traits resolved into clusters. The Journal of Abnormal and Social Psychology, 38, 476-506.

Cattell, R. B. (1963). Theory of fluid and crystallized intelligence: A critical experiment. Journal of Educational Psychology, 54, 1-22.

Chen, F. F., Sousa, K. H., \& West, S. G. (2005). Testing measurement invariance of secondorder factor models. Structural Equation Modeling, 12, 471-492. doi:10.1207/s15328007sem1203_7

Cheung, G. W., \& Rensvold, R. B. (1999). Testing factorial invariance across groups: A reconceptualization and proposed new method. Journal of Management, 25, 1-27.

Cheung, G. W., \& Rensvold, R. B. (2002). Evaluating goodness-of-fit indexes for testing measurement invariance. Structural Equation Modeling, 9, 233-255. 
Claes, L., Smits, D., \& Bijttebier, P. (2014). The Dutch version of the Emotion Reactivity Scale: Validation and relation with various behaviors in a sample of high school students. European Journal of Psychological Assessment, 30, 73-79.

Cleland, C., Magura, S., Foote, J., Rosenblum, A., \& Kosanke, N. (2005). Psychometric properties of the Toronto alexithymia scale (TAS-20) for substance users. Journal of Psychosomatic Research, 58, 299-306.

Cole, P. M., Michel, M. K., \& Teti, L. O. D. (1994). The development of emotion regulation and dysregulation: A clinical perspective. Monographs of the society for research in child development, 59, 73-102.

Comrey, A. L. (1988). Factor-analytic methods of scale development in personality and clinical psychology. Journal of Consulting and Clinical Psychology, 56, 754-761.

Conklin, L. R., Cassiello-Robbins, C., Brake, C. A., Sauer-Zavala, S., Farchione, T. J., Ciraulo, D. A., \& Barlow, D. H. (2015). Relationships among adaptive and maladaptive emotion regulation strategies and psychopathology during the treatment of comorbid anxiety and alcohol use disorders. Behaviour Research and Therapy, 73, 124-130.

Connelly, M., \& Denney, D. R. (2007). Regulation of emotions during experimental stress in alexithymia. Journal of Psychosomatic Research, 62, 649-656.

Consedine, N. S., \& Mauss, I. (2014). Tasks, capacities, and tactics: A skill-based conceptualization of emotion regulation across the lifespan. In P. Verhaeghen \& C. Hertzog (Eds.), The Oxford handbook of emotion, social cognition, and problem solving in adulthood (pp. 142-154). New York, NY, US: Oxford University Press.

Cooper, M. L., Frone, M. R., Russell, M., \& Mudar, P. (1995). Drinking to regulate positive and negative emotions: A motivational model of alcohol use. Journal of Personality and Social Psychology, 69, 990-1005. 
Coriale, G., Bilotta, E., Leone, L., Cosimi, F., Porrari, R., De Rosa, F., \& Ceccanti, M. (2012). Avoidance coping strategies, alexithymia and alcohol abuse: A mediation analysis. Addictive Behaviors, 37, 1224-1229.

Costa, P. T., \& McCrae, R. R. (1992). Normal personality assessment in clinical practice: The NEO Personality Inventory. Psychological Assessment, 4, 5-13.

Costa, P. T., \& McCrae, R. R. (1995). Domains and facets: Hierarchical personality assessment using the Revised NEO Personality Inventory. Journal of Personality Assessment, 64, 21-50.

Costa, P. T., \& McCrae, R. R. (2010). The NEO personality inventory-3. Odessa, FL: Psychological Assessment Resources.

Curran, P. J., West, S. G., \& Finch, J. F. (1996). The robustness of test statistics to nonnormality and specification error in confirmatory factor analysis. Psychological Methods, 1, 16-29.

Czernecka, K., \& Szymura, B. (2008). Alexithymia-imagination-creativity. Personality and Individual Differences, 45, 445-450.

D’Argembeau, A., \& Van der Linden, M. (2006). Individual differences in the phenomenology of mental time travel: The effect of vivid visual imagery and emotion regulation strategies. Consciousness and Cognition, 15, 342-350.

D’Avanzato, C., Joormann, J., Siemer, M., \& Gotlib, I. H. (2013). Emotion regulation in depression and anxiety: examining diagnostic specificity and stability of strategy use. Cognitive Therapy and Research, 37, 968-980.

Davidson, R. J. (1998). Affective style and affective disorders: Perspectives from affective neuroscience. Cognition and Emotion, 12, 307-330. 
Davidson, R. J. (2015). Comment: Affective chronometry has come of age. Emotion Review, 7, 368-370.

Davis, M. H. (1983). Measuring individual differences in empathy: Evidence for a multidimensional approach. Journal of Personality and Social Psychology, 44, $113-$ 126.

De France, K., \& Hollenstein, T. (2017). Assessing emotion regulation repertoires: The regulation of emotion systems survey. Personality and Individual Differences, 119, $204-215$.

de Timary, P., Luts, A., Hers, D., \& Luminet, O. (2008). Absolute and relative stability of alexithymia in alcoholic inpatients undergoing alcohol withdrawal: Relationship to depression and anxiety. Psychiatry Research, 157, 105-113.

Diaz, A., \& Eisenberg, N. (2015). The process of emotion regulation is different from individual differences in emotion regulation: Conceptual arguments and a focus on individual differences. Psychological Inquiry, 26, 37-47.

Duddu, V., Isaac, M. K., \& Chaturvedi, S. K. (2003). Alexithymia in somatoform and depressive disorders. Journal of Psychosomatic Research, 54, 435-438.

du Pont, A., Welker, K. Gilbert, K. E., \& Gruber, J. (2016). The emerging field of positive emotion dysregulation. In K. D. Vohs \& R. F. Baumeister (Eds.), Handbook of selfregulation: Research, theory and applications (pp. 364-379). New York, NY: Guilford Press.

Eastabrook, J. M., Lanteigne, D. M., \& Hollenstein, T. (2013). Decoupling between physiological, self-reported, and expressed emotional responses in alexithymia. Personality and Individual Differences, 55, 978-982. 
Edwards, E. R., \& Wupperman, P. (2017). Emotion regulation mediates effects of alexithymia and emotion differentiation on impulsive aggressive behavior. Deviant Behavior, 38, 1160-1171.

Edwards, E., Shivaji, S., \& Wupperman, P. (2018). The emotion mapping activity: Preliminary evaluation of a mindfulness-informed exercise to improve emotion labeling in alexithymic persons. Scandinavian Journal of Psychology, 1-9. http://dx.doi.org/10.1111/sjop.12438.

Edwards, E. R., \& Wupperman, P. (2017). Emotion regulation mediates effects of alexithymia and emotion differentiation on impulsive aggressive behavior. Deviant Behavior, 38, 1160-1171.

Ehring, T., \& Quack, D. (2010). Emotion regulation difficulties in trauma survivors: The role of trauma type and PTSD symptom severity. Behavior Therapy, 41, 587-598.

Eisenberg, N., Fabes, R. A., Guthrie, I. K., \& Reiser, M. (2000). Dispositional emotionality and regulation: their role in predicting quality of social functioning. Journal of Personality and Social Psychology, 78, 136-157.

Eisenberg, N., Hofer, C., Sulik, M. J., \& Spinrad, T. L. (2014). Self-regulation, effortful control, and their socioemotional correlates. In J. J. Gross (Ed.). Handbook of emotion regulation (pp. 157-172). New York: Guilford Press.

Ekman, P., \& Friesen, W. V. (1971). Constants across cultures in the face and emotion. Journal of Personality and Social Psychology, 17, 124-129.

Ellard, K. K., Fairholme, C. P., Boisseau, C. L., Farchione, T. J., \& Barlow, D. H. (2010). Unified protocol for the transdiagnostic treatment of emotional disorders: Protocol development and initial outcome data. Cognitive and Behavioral Practice, 17, 88101. 
English, T., \& John, O. P. (2013). Understanding the social effects of emotion regulation: The mediating role of authenticity for individual differences in suppression. Emotion, 13, 314-329.

Erni, T., Lötscher, K., \& Modestin, J. (1997). Two-factor solution of the 20-ltem Toronto alexithymia scale confirmed. Psychopathology, 30, 335-340.

Etkin, A., Büchel, C., \& Gross, J. J. (2015). The neural bases of emotion regulation. Nature Reviews Neuroscience, 16, 693-700.

Evers, C., Hopp, H., Gross, J. J., Fischer, A. H., Manstead, A. S., \& Mauss, I. B. (2014). Emotion response coherence: A dual-process perspective. Biological Psychology, 98, $43-49$.

Eysenck, H. J. (1991). Dimensions of personality: 16, 5 or 3?-Criteria for a taxonomic paradigm. Personality and Individual Differences, 12, 773-790.

Fabrigar, L. R., Wegener, D. T., MacCallum, R. C., \& Strahan, E. J. (1999). Evaluating the use of exploratory factor analysis in psychological research. Psychological Methods, 4, 272-299.

Fairholme, C. P., Boisseau, C. L., Ellard, K. K., Ehrenreich, J. T., \& Barlow, D. H. (2010). Emotions, emotion regulation, and psychological treatment: A unified perspective. In A. M. King, \& D. M. Sloan (Eds.). Emotion regulation and psychopathology: A transdiagnostic approach to etiology and treatment (pp. 283-309). New York, NY: Guilford.

Farchione, T. J., Fairholme, C. P., Ellard, K. K., Boisseau, C. L., Thompson-Hollands, J., Carl, J. R., ... \& Barlow, D. H. (2012). Unified protocol for transdiagnostic treatment of emotional disorders: A randomized controlled trial. Behavior Therapy, 43, 666678. 
Fernandes, B. S., Williams, L. M., Steiner, J., Leboyer, M., Carvalho, A. F., \& Berk, M. (2017). The new field of 'precision psychiatry'. BMC medicine, 15, 80.

Folkman, S., \& Lazarus, R. S. (1980). An analysis of coping in a middle-aged community sample. Journal of Health and Social Behavior, 219-239.

Foran, H. M., \& O'Leary, K. D. (2013). The role of relationships in understanding the alexithymia-depression link. European Journal of Personality, 27, 470-480.

Fox, H. C., Axelrod, S. R., Paliwal, P., Sleeper, J., \& Sinha, R. (2007). Difficulties in emotion regulation and impulse control during cocaine abstinence. Drug and Alcohol Dependence, 89, 298-301.

Fox, H. C., Hong, K. A., \& Sinha, R. (2008). Difficulties in emotion regulation and impulse control in recently abstinent alcoholics compared with social drinkers. Addictive Behaviors, 33, 388-394.

Fraley, R. C., Heffernan, M. E., Vicary, A. M., \& Brumbaugh, C. C. (2011). The Experiences in Close Relationships_-Relationship Structures Questionnaire: A method for assessing attachment orientations across relationships. Psychological Assessment, 23, $615-625$.

Friedlander, L., Lumley, M. A., Farchione, T., \& Doyal, G. (1997). Testing the alexithymia hypothesis: Physiological and subjective responses during relaxation and stress. The Journal of Nervous and Mental Disease, 185, 233-239.

Fukunishi, I., Yoshida, H., \& Wogan, J. (1998). Development of the alexithymia scale for children: A preliminary study. Psychological Reports, 82, 43-49.

Garnefski, N., \& Kraaij, V. (2007). The cognitive emotion regulation questionnaire. European Journal of Psychological Assessment, 23, 141-149. 
Gerbing, D. W., \& Anderson, J. C. (1984). On the meaning of within-factor correlated measurement errors. Journal of Consumer Research, 11, 572-580.

Gerbing, D. W., \& Anderson, J. C. (1988). An updated paradigm for scale development incorporating unidimensionality and its assessment. Journal of Marketing Research, $25,186-192$.

Gignac, G. E., Palmer, B. R., \& Stough, C. (2007). A confirmatory factor analytic investigation of the TAS-20: Corroboration of a five-factor model and suggestions for improvement. Journal of Personality Assessment, 89, 247-257.

Gilbert, K. E., Nolen-Hoeksema, S., \& Gruber, J. (2013). Positive emotion dysregulation across mood disorders: How amplifying versus dampening predicts emotional reactivity and illness course. Behaviour Research and Therapy, 51, 736-741.

Giuliani, N. R., \& Berkman, E. T. (2015). Craving is an affective state and its regulation can be understood in terms of the extended process model of emotion regulation. Psychological Inquiry, 26, 48-53.

Glenn, C. R., \& Klonsky, E. D. (2009). Emotion dysregulation as a core feature of borderline personality disorder. Journal of Personality Disorders, 23, 20-28.

Gold, M. S., \& Bentler, P. M. (2000). Treatments of missing data: A Monte Carlo comparison of RBHDI, iterative stochastic regression imputation, and expectation maximization. Structural Equation Modeling, 7, 319-355.

Goldberg, D. P., Krueger, R. F., Andrews, G., \& Hobbs, M. J. (2009). Emotional disorders: Cluster 4 of the proposed meta-structure for DSM-V and ICD-11: Paper 5 of 7 of the thematic section: 'A proposal for a meta-structure for DSM-V and ICD11'. Psychological Medicine, 39, 2043-2059. 
Goldin, P. R., \& Gross, J. J. (2010). Effects of mindfulness-based stress reduction (MBSR) on emotion regulation in social anxiety disorder. Emotion, 10, 83-91.

Goleman, D., Boyatzis, R., \& McKee, A. (2013). Primal leadership: Realizing the power of emotional intelligence. Boston, MA, US: Harvard Business School Press.

Golena, N. (2014). The relation between alexithymia and the vividness of memories during the recall of sad memories (Bachelor's thesis). Enschede, Netherlands: University of Twente.

Gori, A., Giannini, M., Palmieri, G., Salvini, R., \& Schuldberg, D. (2012). Assessment of alexithymia: Pychometric properties of the psychological treatment inventoryalexithymia scale (PTI-AS). Psychology, 3, 231-236.

Gorsuch, R. L. (1983). Factor analysis. Hillsdale, NJ: Erlbaum.

Grabe, H. J., Löbel, S., Dittrich, D., Bagby, R. M., Taylor, G. J., Quilty, L. C., \& Freyberger, H. J. (2009). The German version of the Toronto Structured Interview for Alexithymia: Factor structure, reliability, and concurrent validity in a psychiatric patient sample. Comprehensive Psychiatry, 50, 424-430.

Grandey, A. A. (2000). Emotional regulation in the workplace: A new way to conceptualize emotional labor. Journal of Occupational Health Psychology, 5, 95-110.

Gratz, K. L., \& Roemer, L. (2004). Multidimensional assessment of emotion regulation and dysregulation: Development, factor structure, and initial validation of the difficulties in emotion regulation scale. Journal of Psychopathology and Behavioral Assessment, $26,41-54$.

Greenberg, L. S., \& Paivio, S. C. (2003). Working with emotions in psychotherapy. Guilford Press.

Gross, J. J. (1998). The emerging field of emotion regulation: An integrative review. Review of General Psychology, 2, 271-299. 
Gross, J. J. (2014). Emotion regulation: Conceptual and empirical foundations. In J. J. Gross (Ed.), Handbook of emotion regulation (pp. 3-20). New York, NY: Guilford Press.

Gross, J. J. (2015a). Emotion regulation: Current status and future prospects. Psychological Inquiry, 26, 1-26.

Gross, J. J. (2015b). The extended process model of emotion regulation: Elaborations, applications, and future directions. Psychological Inquiry, 26, 130-137.

Gross, J. J., \& Barrett, L. F. (2011). Emotion generation and emotion regulation: One or two depends on your point of view. Emotion Review, 3, 8-16.

Gross, J. J., \& Jazaieri, H. (2014). Emotion, emotion regulation, and psychopathology: An affective science perspective. Clinical Psychological Science, 2, 387-401.

Gross, J. J., \& John, O. P. (2003). Individual differences in two emotion regulation processes: Implications for affect, relationships, and well-being. Journal of Personality and Social Psychology, 85, 348-362.

Grossmann, I., Karasawa, M., Kan, C., \& Kitayama, S. (2014). A cultural perspective on emotional experiences across the life span. Emotion, 14, 679-692.

Groth-Marnat, G. (2009). Handbook of psychological assessment. New York, NY: Wiley.

Gruber, J. (2011). Can feeling too good be bad? Positive emotion persistence (PEP) in bipolar disorder. Current Directions in Psychological Science, 20, 217-221.

Gruber, J., Eidelman, P., Johnson, S. L., Smith, B., \& Harvey, A. G. (2011). Hooked on a feeling: Rumination about positive and negative emotion in inter-episode bipolar disorder. Journal of Abnormal Psychology, 120, 956-961.

Gruber, J., Harvey, A. G., \& Gross, J. J. (2012). When trying is not enough: Emotion regulation and the effort-success gap in bipolar disorder. Emotion, 12, 997-1003. 
Gruber, J., Harvey, A. G., \& Purcell, A. (2011). What goes up can come down? A preliminary investigation of emotion reactivity and emotion recovery in bipolar disorder. Journal of Affective Disorders, 133, 457-466.

Gruber, J., Johnson, S. L., Oveis, C., \& Keltner, D. (2008). Risk for mania and positive emotional responding: Too much of a good thing? Emotion, 8, 23-33.

Grynberg, D., Luminet, O., Corneille, O., Grèzes, J., \& Berthoz, S. (2010). Alexithymia in the interpersonal domain: A general deficit of empathy? Personality and Individual Differences, 49, 845-850.

Gullone, E., \& Taffe, J. (2012). The Emotion Regulation Questionnaire for Children and Adolescents (ERQ-CA): A psychometric evaluation. Psychological Assessment, 24, 409-417.

Han, K., Burns, G. N., Weed, N. C., Hatchett, G. T., \& Kurokawa, N. K. (2009). Evaluation of an observer form of the Coping Inventory for Stressful Situations. Educational and Psychological Measurement, 69, 675-695.

Harre, R. (1986). The social constructionist viewpoint. In R. Harre (Ed.), The social construction of emotions (pp. 2-14). Oxford, UK: Blackwell.

Harris, R. (2009). ACT made simple: An easy-to-read primer on acceptance and commitment therapy. New Harbinger Publications.

Haviland, M. G., Hendryx, M. S., Cummings, M. A., Shaw, D. G., \& MacMurray, J. P. (1991). Multidimensionality and state dependency of alexithymia in recently sober alcoholics. The Journal of Nervous and Mental Disease, 179, 284-290.

Haviland, M. G., \& Reise, S. P. (1996). A California Q-set alexithymia prototype and its relationship to ego-control and ego-resiliency. Journal of Psychosomatic Research, $41,597-607$. 
Haviland, M. G., \& Reise, S. P. (1996). Structure of the twenty-item Toronto alexithymia scale. Journal of Personality Assessment, 66, 116-125.

Haviland, M. G., Shaw, D. G., MacMurray, J. P., \& Cummings, M. A. (1988). Validation of the Toronto Alexithymia Scale with substance abusers. Psychotherapy and Psychosomatics, 50, 81-87.

Haviland, M. G., Warren, W. L., \& Riggs, M. L. (2000). An observer scale to measure alexithymia. Psychosomatics, 41, 385-392.

Hayes, S. C., Strosahl, K. D., \& Wilson, K. G. (1999). Acceptance and commitment therapy. New York: Guilford Press.

Henry, C., Van den Bulke, D., Bellivier, F., Roy, I., Swendsen, J., M’Bailara, K., ... Leboyer, M. (2008). Affective lability and affect intensity as core dimensions of bipolar disorders during euthymic period. Psychiatry Research, 159, 1-6.

Honkalampi, K., Hintikka, J., Laukkanen, E., \& Viinamäki, J. L. H. (2001). Alexithymia and depression: A prospective study of patients with major depressive disorder. Psychosomatics, 42, 229-234.

Harrison, A., Sullivan, S., Tchanturia, K., \& Treasure, J. (2010). Emotional functioning in eating disorders: attentional bias, emotion recognition and emotion regulation. Psychological Medicine, 40, 1887-1897.

Hogan, R., \& Nicholson, R. A. (1988). The meaning of personality test scores. American Psychologist, 43, 621-626.

Hu, L. T., \& Bentler, P. M. (1998). Fit indices in covariance structure modeling: Sensitivity to underparameterized model misspecification. Psychological Methods, 3, 424-453.

Inslegers, R., Meganck, R., Ooms, E., Vanheule, S., Taylor, G., Bagby, R. M., ... Desmet, M. (2013). The Dutch language version of the Toronto structured interview for 
alexithymia: Reliability, factor structure and concurrent validity. Acta Psychiatrica Belgica, 53, 93-116.

Izadpanah, S., Barnow, S., Neubauer, A. B., \& Holl, J. (2017). Development and validation of the Heidelberg Form for Emotion Regulation Strategies (HFERST): Factor structure, reliability, and validity. Assessment, 1-27. doi: 1073191117720283

John, O. P., \& Eng, J. (2014). Three approaches to individual differences in affect regulation: Conceptualizations, measures, and findings. In J. J. Gross (Ed.). Handbook of emotion regulation (pp. 321-345). (2nd ed.). New York, NY: Guilford.

John, O. P., \& Gross, J. J. (2004). Healthy and unhealthy emotion regulation: Personality processes, individual differences, and life span development. Journal of Personality, 72, 1301-1334.

Joormann, J., \& Gotlib, I. H. (2010). Emotion regulation in depression: Relation to cognitive inhibition. Cognition and Emotion, 24, 281-298.

Jørgensen, M. M., Zachariae, R., Skytthe, A., \& Kyvik, K. (2007). Genetic and environmental factors in alexithymia: A population-based study of 8,785 Danish twin pairs. Psychotherapy and Psychosomatics, 76, 369-375.

Kennedy, M., \& Franklin, J. (2002). Skills-based treatment for alexithymia: An exploratory case series. Behaviour Change, 19, 158-171.

Kessler, R. C., Chiu, W. T., Demler, O., \& Walters, E. E. (2005). Prevalence, severity, and comorbidity of 12-month DSM-IV disorders in the National Comorbidity Survey Replication. Archives of General Psychiatry, 62, 617-627.

Khantzian, E. J. (1997). The self-medication hypothesis of substance use disorders: A reconsideration and recent applications. Harvard Review of Psychiatry, 4, 231-244. 
Kia-Keating, M., No, U., Moore, S., Furlong, M. J., Liu, S., \& You, S. (2017). Structural validity of the Depression, Anxiety, and Stress Scales-21 adapted for US undergraduates. Emerging Adulthood, 1-7.

Kim, S. H., \& Hamann, S. (2007). Neural correlates of positive and negative emotion regulation. Journal of Cognitive Neuroscience, 19, 776-798.

Kline, P. (1979). Psychometrics and psychology. London: Academic Press.

Kline, P. (2013). Handbook of psychological testing. Routledge.

Kline, R. B. (2005). Principles and practice of structural equation modeling. New York: The Guilford Press.

Koch, A. S., Kleiman, A., Wegener, I., Zur, B., Imbierowicz, K., Geiser, F., \& Conrad, R. (2015). Factorial structure of the 20 -item Toronto alexithymia scale in a large sample of somatoform patients. Psychiatry Research, 225, 355-363.

Kooiman, C. G., Spinhoven, P., \& Trijsburg, R.W. (2002). The assessment of alexithymia: A critical review of the literature and a psychometric study of the Toronto Alexithymia Scale-20. Journal of Psychosomatic Research, 53, 1083-1090.

Kring, A. M., \& Bachorowski, J. A. (1999). Emotions and psychopathology. Cognition and Emotion, 13, 575-599.

Kuppens, P., \& Verduyn, P. (2015). Looking at emotion regulation through the window of emotion dynamics. Psychological Inquiry, 26, 72-79.

Lane, R. D., \& Schwartz, G. E. (1987). Levels of emotional awareness: A cognitivedevelopmental theory and its application to psychopathology. The American Journal of Psychiatry, 144, 133-143.

Lane, R. D., Lee, S., Reidel, R., Weldon, V., Kaszniak, A., \& Schwartz, G. E. (1996). Impaired verbal and nonverbal emotion recognition in alexithymia. Psychosomatic Medicine, 58, 203-210. 
Lane, R. D., Quinlan, D. M., Schwartz, G. E., Walker, P. A., \& Zeitlin, S. B. (1990). The levels of emotional awareness scale: A cognitive-developmental measure of emotion. Journal of Personality Assessment, 55, 124-134.

Lane, R. D., Weihs, K. L., Herring, A., Hishaw, A., \& Smith, R. (2015). Affective agnosia: Expansion of the alexithymia construct and a new opportunity to integrate and extend Freud's legacy. Neuroscience \& Biobehavioral Reviews, 55, 594-611.

Lannoy, S., Heeren, A., Rochat, L., Rossignol, M., Van der Linden, M., \& Billieux, J. (2014). Is there an all-embracing construct of emotion reactivity? Adaptation and validation of the emotion reactivity scale among a French-speaking community sample. Comprehensive Psychiatry, 55, 1960-1967.

Larsen, R. J. (2000). Toward a science of mood regulation. Psychological Inquiry, 11, 129141.

Lazarus, R. S. (1991). Emotion and adaptation. New York, NY: Oxford University Press.

Lee, D. J., Witte, T. K., Bardeen, J. R., Davis, M. T., \& Weathers, F. W. (2016). A factor analytic evaluation of the difficulties in emotion regulation scale. Journal of Clinical Psychology, 72, 933-946.

Leising, D., Grande, T., \& Faber, R. (2009). The Toronto Alexithymia Scale (TAS-20): A measure of general psychological distress. Journal of Research in Personality, 43, $707-710$.

Levy-Gigi, E., Bonanno, G. A., Shapiro, A. R., Richter-Levin, G., Kéri, S., \& Sheppes, G. (2016). Emotion regulatory flexibility sheds light on the elusive relationship between repeated traumatic exposure and posttraumatic stress disorder symptoms. Clinical Psychological Science: A Journal of the Association for Psychological Science, 4, 2839. 
Leweke, F., Bausch, S., Leichsenring, F., Walter, B., \& Stingl, M. (2009). Alexithymia as a predictor of outcome of psychodynamically oriented inpatient treatment. Psychotherapy Research, 19, 323-331.

Leweke, F., Leichsenring, F., Kruse, J., \& Hermes, S. (2012). Is alexithymia associated with specific mental disorders. Psychopathology, 45, 22-28.

Lezak, M. D., Howieson, D. B., \& Loring, D. W. (2004). Neuropsychological assessment. USA: Oxford University Press.

Li, S., Zhang, B., Guo, Y., \& Zhang, J. (2015). The association between alexithymia as assessed by the 20-item Toronto Alexithymia Scale and depression: A meta-analysis. Psychiatry Research, 227, 1-9.

Linehan, M. (1993). Cognitive-behavioral treatment of borderline personality disorder. New York, NY: Guilford Press.

Little, T. D., Lindenberger, U., \& Nesselroade, J. R. (1999). On selecting indicators for multivariate measurement and modeling with latent variables: When "good" indicators are bad and "bad" indicators are good. Psychological Methods, 4, 192-211.

Larsen, R. J., \& Diener, E. (1987). Affect intensity as an individual difference characteristic: A review. Journal of Research in Personality, 21, 1-39.

Loas, G., Corcos, M., Stephan, P., Pellet, J., Bizouard, P., Venisse, J. L., et al. (2001). Factorial structure of the 20-item Toronto alexithymia scale: Confirmatory factorial analyses in nonclinical and clinical samples. Journal of Psychosomatic Research, 50, $255-261$.

Loas, G., Otmani, O., Verrier, A., Fremaux, D., \& Marchand, M. P. (1996). Factor analysis of the French version of the 20-ltem Toronto alexithymia scale (TAS-20). Psychopathology, 29, 139-144. 
Lovibond, P. F., \& Lovibond, S. H. (1995). The structure of negative emotional states: Comparison of the Depression Anxiety Stress Scales (DASS) with the Beck Depression and Anxiety Inventories. Behaviour Research and Therapy, 33, 335-343.

Luminet, O., Bagby, R. M., \& Taylor, G. J. (2001). An evaluation of the absolute and relative stability of alexithymia in patients with major depression. Psychotherapy and Psychosomatics, 70, 254-260.

Luminet, O., Rimé, B., Bagby, R. M., \& Taylor, G. (2004). A multimodal investigation of emotional responding in alexithymia. Cognition and Emotion, 18, 741-766.

Luminet, O., Rokbani, L., Ogez, D., \& Jadoulle, V. (2007). An evaluation of the absolute and relative stability of alexithymia in women with breast cancer. Journal of Psychosomatic Research, 62, 641-648.

Luminet, O., Vermeulen, N., Demaret, C., Taylor, G. J., \& Bagby, R. M. (2006). Alexithymia and levels of processing: Evidence for an overall deficit in remembering emotion words. Journal of Research in Personality, 40, 713-733.

Lundh, L. G., \& Simonsson-Sarnecki, M. (2001). Alexithymia, emotion, and somatic complaints. Journal of Personality, 69, 483-510.

Lundh, L. G., Johnsson, A., Sundqvist, K., \& Olsson, H. (2002). Alexithymia, memory of emotion, emotional awareness, and perfectionism. Emotion, 2, 361-379.

Lyvers, M., McCann, K., Coundouris, S., Edwards, M. S., \& Thorberg, F. A. (2018). Alexithymia in relation to alcohol use, emotion recognition, and empathy: The role of externally oriented thinking. American Journal of Psychology, 131, 41-51.

Mantani, T., Okamoto, Y., Shirao, N., Okada, G., \& Yamawaki, S. (2005). Reduced activation of posterior cingulate cortex during imagery in subjects with high degrees of alexithymia: A functional magnetic resonance imaging study. Biological Psychiatry, 57, 982-990. 
Marchesi, C., Ossola, P., Tonna, M., \& De Panfilis, C. (2014). The TAS-20 more likely measures negative affects rather than alexithymia itself in patients with major depression, panic disorder, eating disorders and substance use disorders. Comprehensive Psychiatry, 55, 972-978.

Markus, H. (1977). Self-schemata and processing information about the self. Journal of Personality and Social Psychology, 35, 63-78.

Marsh, H. W., \& Hocevar, D. (1985). Application of confirmatory factor analysis to the study of self-concept: First-and higher order factor models and their invariance across groups. Psychological Bulletin, 97, 562-582.

Marsh, H. W., Hau, K. T., \& Wen, Z. (2004). In search of golden rules: Comment on hypothesis-testing approaches to setting cutoff values for fit indexes and dangers in overgeneralizing Hu and Bentler's (1999) findings. Structural Equation Modeling, 11, $320-341$.

Marty, P., \& de M'Uzan, M. (1963). La “pensee operatoire”. Revue Française de Psychanalyse, 27, 1345-1356.

Matsumoto, D., Yoo, S. H., \& Nakagawa, S. (2008). Culture, emotion regulation, and adjustment. Journal of Personality and Social Psychology, 94, 925-937.

Matsunaga, M. (2010). How to factor-analyze your data right: Do's, don'ts, and how-to's. International Journal of Psychological Research, 3, 97-110.

Mattila, A. K., Keefer, K. V., Taylor, G. J., Joukamaa, M., Jula, A., Parker, J. D., \& Bagby, R. M. (2010). Taxometric analysis of alexithymia in a general population sample from Finland. Personality and Individual Differences, 49, 216-221.

Mauss, I. B., Cook, C. L., Cheng, J. Y., \& Gross, J. J. (2007). Individual differences in cognitive reappraisal: Experiential and physiological responses to an anger provocation. International Journal of Psychophysiology, 66, 116-124. 
Mauss, I. B., Levenson, R. W., McCarter, L., Wilhelm, F. H., \& Gross, J. J. (2005). The tie that binds? Coherence among emotion experience, behavior, and physiology. Emotion, 5, 175-190.

Mayer, J. D., Salovey, P., \& Caruso, D. (2002). Mayer-Salovey-Caruso Emotional Intelligence Test (MSCEIT): User's manual. Toronto, ON: Multi-Health Systems.

McDougall, J. (1974). The psychosomata and the psychoanalytic process. Internal Review of Psychoanalysis, 1, 437-459.

McGillivray, L., Becerra, R., \& Harms, C. (2017). Prevalence and demographic correlates of alexithymia: A comparison between Australian psychiatric and community samples. Journal of Clinical Psychology, 73, 76-87.

McGrew, K. S. (2009). CHC theory and the human cognitive abilities project: Standing on the shoulders of the giants of psychometric intelligence research. Intelligence, 37, 110.

McHugh, R. K., Reynolds, E. K., Leyro, T. M., \& Otto, M. W. (2013). An examination of the association of distress intolerance and emotion regulation with avoidance. Cognitive Therapy and Research, 37, 363-367.

Meganck, R., Vanheule, S., \& Desmet, M. (2008). Factorial validity and measurement invariance of the 20-itemToronto Alexithymia Scale in clinical and nonclinical samples. Assessment, 15, 36-47.

Melka, S. E., Lancaster, S. L., Bryant, A. R., \& Rodriguez, B. F. (2011). Confirmatory factor and measurement invariance analyses of the Emotion Regulation Questionnaire. Journal of Clinical Psychology, 67, 1283-1293.

Mennin, D. S., \& Farach, F. J. (2007). Emotion and evolving treatments for adult psychopathology. Clinical Psychology: Science and Practice, 14, 329-352. 
Mennin, D. S., \& Fresco, D. M. (2014). Emotion regulation therapy. In J. J. Gross (Ed.), Handbook of emotion regulation (pp. 469-490). New York, NY, US: Guilford Press.

Montag, C., \& Panksepp, J. (2017). Primary emotional systems and personality: an evolutionary perspective. Frontiers in Psychology, 8, 464.

Moore, S. A., Zoellner, L. A., \& Mollenholt, N. (2008). Are expressive suppression and cognitive reappraisal associated with stress-related symptoms?. Behaviour Research and Therapy, 46, 993-1000.

Morera, O. F., Culhane, S. E.,Watson, P. J., \& Skewes, M. C. (2005). Assessing the reliability and validity of the Bermond-Vorst Alexithymia Questionnaire among US Anglo and US Hispanic samples. Journal of Psychosomatic Research, 58, 289-298.

Müller, J., Bühner, M., \& Ellgring, H. (2003). Is there a reliable factorial structure in the 20item Toronto alexithymia scale?: A comparison of factor models in clinical and normal adult samples. Journal of Psychosomatic Research, 55, 561-568.

Müller, J., Bühner, M., \& Ellgring, H. (2004). The assessment of alexithymia: Psychometric properties and validity of the Bermond-Vorst alexithymia questionnaire. Personality and Individual Differences, 37, 373-391.

Nelis, D., Quoidbach, J., Hansenne, M., \& Mikolajczak, M. (2011). Measuring individual differences in emotion regulation: The emotion regulation profile-revised (ERP-R). Psychologica Belgica, 51, 49-91.

Nemiah, J. C. (1977). Alexithymia: Theoretical considerations. Psychotherapy and Psychosomatics, 28, 199-206. 
Nemiah, J. C. (1984). The psychodynamic view of anxiety. In R. O. Pasnau (Ed.), Diagnosis and treatment of anxiety disorders (pp. 117-137). Washington, DC: American Psychiatric Press.

Nemiah, J. C., \& Sifneos, P. E. (1970). Psychosomatic illness: A problem in communication. Psychotherapy and Psychosomatics, 18, 154-160.

Neumann, D., Malec, J. F., \& Hammond, F. M. (2017). Reductions in alexithymia and emotion dysregulation after training emotional self-awareness following traumatic brain injury: A phase I trial. The Journal of Head Trauma Rehabilitation, 32, 286295.

New, A. S., Rot, M. A. H., Ripoll, L. H., Perez-Rodriguez, M. M., Lazarus, S., Zipursky, E., ...Siever, L. J. (2012). Empathy and alexithymia in borderline personality disorder: Clinical and laboratory measures. Journal of Personality Disorders, 26, 660-675.

Newton, T. L., \& Contrada, R. J. (1994). Alexithymia and repression: Contrasting emotion focused coping styles. Psychosomatic Medicine, 56, 457-462.

Nock, M. K., Wedig, M. M., Holmberg, E. B., \& Hooley, J. M. (2008). The emotion reactivity scale: Development, evaluation, and relation to self injurious thoughts and behaviors. Behavior Therapy, 39, 107-116.

Nolen-Hoeksema, S., \& Watkins, E. R. (2011). A heuristic for developing transdiagnostic models of psychopathology: Explaining multifinality and divergent trajectories. Perspectives on Psychological Science, 6, 589-609.

Norcross, J. C., \& Karpiak, C. P. (2012). Clinical psychologists in the 2010s: 50 years of the APA division of clinical psychology. Clinical Psychology: Science and Practice, 19, 1-12.

Nunnally, J. C. (1978). Psychometric Methods. New York: McGraw Hill.

Nunnally, J. C., \& Bernstein, I. H. (1994). Psychometric theory. New York: McGraw-Hill. 
Ochsner, K. N., \& Gross, J. J. (2014). The neural bases of emotion and emotion regulation: A valuation perspective. In J. J. Gross (Ed.). Handbook of emotion regulation (pp. 2342). New York, NY: Guilford Press.

Osborne, T. L., Michonski, J., Sayrs, J., Welch, S. S., \& Anderson, L. K. (2017). Factor structure of the Difficulties in Emotion Regulation Scale (DERS) in adult outpatients receiving dialectical behavior therapy (DBT). Journal of Psychopathology and Behavioral Assessment, 39, 355-371.

Panayiotou, G., Leonidou, C., Constantinou, E., Hart, J., Rinehart, K. L., Sy, J. T., \& Björgvinsson, T. (2015). Do alexithymic individuals avoid their feelings? Experiential avoidance mediates the association between alexithymia, psychosomatic, and depressive symptoms in a community and a clinical sample. Comprehensive Psychiatry, 56, 206-216.

Pandey, R., Mandal, M. K., Taylor, G. J., \& Parker, J. D. (1996). Crosscultural alexithymia: Development and validation of a Hindi translation of the 20-item Toronto alexithymia scale. Journal of Clinical Psychology, 52, 173-176.

Panksepp, J. (1998). Affective neuroscience: The foundations of human and animal emotions. New York, NY: Oxford University Press.

Panksepp, J. (2005). Affective consciousness: Core emotional feelings in animals and humans. Consciousness and Cognition, 14, 30-80.

Parker, J. D., Eastabrook, J. M., Keefer, K. V., \& Wood, L. M. (2010). Can alexithymia be assessed in adolescents? Psychometric properties of the 20-item Toronto alexithymia scale in younger, middle, and older adolescents. Psychological Assessment, 22, 798808. 
Parker, J. D., Keefer, K. V., Taylor, G. J., \& Bagby, R. M. (2008). Latent structure of the alexithymia construct: A taxometric investigation. Psychological Assessment, 20, $385-396$.

Parker, J. D., Taylor, G. J., \& Bagby, R. M. (2003). The 20-item Toronto alexithymia scale:

III. Reliability and factorial validity in a community population. Journal of Psychosomatic Research, 55, 269-275.

Perez, C. R., \& Soto, J. A. (2011). Cognitive reappraisal in the context of oppression: Implications for psychological functioning. Emotion, 11, 675-680.

Piaget, J. (1981). Intelligence and affectivity: Their relationship during child development. Palo Alto, CA: Annual Reviews.

Taylor, G. J., \& Bagby, R.M. (2004). New trends in alexithymia research. Psychotherapy and Psychosomatics, 73, 68-77.

Podsakoff, P. M., MacKenzie, S. B., Lee, J. Y., \& Podsakoff, N. P. (2003). Common method biases in behavioral research: A critical review of the literature and recommended remedies. Journal of Applied Psychology, 88, 879-903.

Pollatos, O., Werner, N. S., Duschek, S., Schandry, R., Matthias, E., Traut-Mattausch, E., \& Herbert, B.M. (2011). Differential effects of alexithymia subscales on autonomic reactivity and anxiety during social stress. Journal of Psychosomatic Research, 70, $525-533$.

Porcelli, P., \& Mihura, J. L. (2010). Assessment of alexithymia with the Rorschach comprehensive system: The Rorschach alexithymia scale (RAS). Journal of Personality Assessment, 92, 128-136.

Preece, D., Becerra, R., \& Campitelli, G. (2018). Assessing emotional reactivity: Psychometric properties of the Perth Emotional Reactivity Scale and the development 
of a short form. Journal of Personality Assessment, 1-9. https://doi.org/10.1080/00223891.2018.1465430.

Preece, D., Becerra, R., Allan, A., Robinson, K., \& Dandy, J. (2017). Establishing the theoretical components of alexithymia via factor analysis: Introduction and validation of the attention-appraisal model of alexithymia. Personality and Individual Differences, 119, 341-352.

Preece, D., Becerra, R., Robinson, K., \& Dandy, J. (2018). Assessing alexithymia: Psychometric properties and factorial invariance of the 20-item Toronto Alexithymia Scale (TAS-20) in nonclinical and psychiatric samples. Journal of Psychopathology and Behavioral Assessment, 40, 276-287.

Preece, D., Becerra, R., Robinson, K., Dandy, J., \& Allan, A. (2018a). The psychometric assessment of alexithymia: Development and validation of the Perth Alexithymia Questionnaire. Personality and Individual Differences, 132, 32-44.

Preece, D.A., Becerra, R., Robinson, K., Dandy, J., \& Allan, A. (2018b). Measuring emotion regulation ability across negative and positive emotions: The Perth Emotion Regulation Competency Inventory (PERCI). Personality and Individual Differences, $135,229-241$.

Preece, D. A., Becerra, R., Robinson, K., \& Gross, J. J. (2019). The Emotion Regulation Questionnaire: Psychometric properties in general community samples. Journal of Personality Assessment, 1-9. http://doi.org/10.1080/00223891.2018.156319

Preston, C. C., \& Colman, A. M. (2000). Optimal number of response categories in rating scales: Reliability, validity, discriminating power, and respondent preferences. Acta Psychologica, 104, 1-15.

Qualtrics. (2014). ESOMAR 28. Retrieved from https://success.qualtrics.com/rs/qualtrics/images/ESOMAR\%2028\%202014.pdf 
Quoidbach, J., Berry, E. V., Hansenne, M., \& Mikolajczak, M. (2010). Positive emotion regulation and well-being: Comparing the impact of eight savoring and dampening strategies. Personality and Individual Differences, 49, 368-373.

Raubenheimer, J. (2004). An item selection procedure to maximise scale reliability and validity. SA Journal of Industrial Psychology, 30, 59-64.

Reise, S. P. (2012). The rediscovery of bifactor measurement models. Multivariate Behavioral Research, 47, 667-696.

Reise, S. P., Moore, T. M., \& Haviland, M. G. (2010). Bifactor models and rotations: Exploring the extent to which multidimensional data yield univocal scale scores. Journal of Personality Assessment, 92, 544-559.

Rieffe, C., Oosterveld, P., Miers, A. C., Terwogt, M. M., \& Ly, V. (2008). Emotion awareness and internalising symptoms in children and adolescents: The emotion awareness questionnaire revised. Personality and Individual Differences, 45, 756761.

Ripper, C. A., Boyes, M. E., Clarke, P. J., \& Hasking, P. A. (2018). Emotional reactivity, intensity, and perseveration: Independent dimensions of trait affect and associations with depression, anxiety, and stress symptoms. Personality and Individual Differences, 121, 93-99.

Rodebaugh, T. L., Woods, C. M., \& Heimberg, R. G. (2007). The reverse of social anxiety is not always the opposite: The reverse-scored items of the social interaction anxiety scale do not belong. Behavior Therapy, 38, 192-206.

Rosenberg, N., Rufer, M., Lichev, V., Ihme, K., Grabe, H. J., Kugel, H., ... Suslow, T. (2016). Observer-rated alexithymia and its relationshipwith the five-factor-model of personality. Acta Psychiatrica Belgica, 56, 118-134. 
Rosenthal, M. Z., Gratz, K. L., Kosson, D. S., Cheavens, J. S., Lejuez, C. W., \& Lynch, T. R. (2008). Borderline personality disorder and emotional responding: A review of the research literature. Clinical Psychology Review, 28, 75-91.

Rottenberg, J. E., \& Johnson, S. L. (2007). Emotion and psychopathology: Bridging affective and clinical science. New York, NY: Guilford Press.

Rottenberg, J., Gross, J. J., \& Gotlib, I. H. (2005). Emotion context insensitivity in major depressive disorder. Journal of Abnormal Psychology, 114, 627-639.

Sala, M. N., Molina, P., Abler, B., Kessler, H., Vanbrabant, L., \& van de Schoot, R. (2012). Measurement invariance of the Emotion Regulation Questionnaire (ERQ). A crossnational validity study. European Journal of Developmental Psychology, 9, 751-757.

Salovey, P., Mayer, J. D., Goldman, S. L., Turvey, C., \& Palfai, T. P. (1995). Emotional attention, clarity, and repair: Exploring emotional intelligence using the Trait MetaMood Scale. In J. W. Pennebaker (Ed.). Emotion, disclosure, and health (pp. 125154). Washington, DC: American Psychological Association.

Samur, D., Tops, M., Schlinkert, C., Quirin, M., Cuijpers, P., \& Koole, S. L. (2013). Four decades of research on alexithymia: Moving toward clinical applications. Frontiers in Psychology, 4, 1-4.

Satorra, A., \& Bentler, P. M. (1994). Corrections to test statistics and standard errors in covariance structure analysis. In A. von Eye \& C. C. Clogg (Eds.), Latent variables analysis: Applications for developmental research (pp. 399-419). Thousand Oaks, CA: Sage Publications, Inc.

Satorra, A., \& Bentler, P. M. (1994). Corrections to test statistics and standard errors in covariance structure analysis. In A. von Eye \& C. C. Clogg (Eds.), Latent variables 
analysis: Applications for developmental research (pp. 399-419). Thousand Oaks, CA, US: Sage Publications, Inc.

Sauer, S., \& Baer, R. (2009). Relationships between thought suppression and symptoms of borderline personality disorder. Journal of Personality Disorders, 23, 48-61.

Sauer, S. E., \& Baer, R. A. (2010). Validation of measures of biosocial precursors to borderline personality disorder: Childhood emotional vulnerability and environmental invalidation. Assessment, 17, 454-466.

Sauer-Zavala, S., \& Barlow, D. H. (2014). The case for borderline personality disorder as an emotional disorder: Implications for treatment. Clinical Psychology: Science and Practice, 21, 118-138.

Sauer-Zavala, S., Boswell, J. F., Gallagher, M. W., Bentley, K. H., Ametaj, A., \& Barlow, D. H. (2012). The role of negative affectivity and negative reactivity to emotions in predicting outcomes in the unified protocol for the transdiagnostic treatment of emotional disorders. Behaviour Research and Therapy, 50, 551-557.

Sauer-Zavala, S., Cassiello-Robbins, C., Ametaj, A. A., Wilner, J. G., \& Pagan, D. (2018). Transdiagnostic treatment personalization: The feasibility of ordering unified protocol modules according to patient strengths and weaknesses. Behavior Modification, 1-26. doi: $10.1177 / 0145445518774914$

Sauer-Zavala, S., Gutner, C. A., Farchione, T. J., Boettcher, H. T., Bullis, J. R., \& Barlow, D. H. (2017). Current definitions of "transdiagnostic" in treatment development: A search for consensus. Behavior Therapy, 48, 128-138. 
Schmader, T., \& Mendes, W. B. (2015). Putting feelings in a social context: Three case studies applying Gross's extended model of emotion regulation. Psychological Inquiry, 26, 116-122.

Schumacker, R. E., \& Lomax, R. G. (2004). A beginner's guide to structural equation modeling. Mahwah, NJ: Erlbaum and Associates.

Sekely, A., Taylor, G. J., \& Bagby, R. M. (2018). Developing a short version of the Toronto structured interview for alexithymia using item response theory. Psychiatry Research, $266,218-227$.

Shaver, P. R., \& Mikulincer, M. (2014). Adult attachment and emotion regulation. In J. Gross (Ed.). Handbook of emotion regulation. New York, NY: Guilford Press.

Sifneos, P. E. (1973). The prevalence of 'alexithymic' characteristics in psychosomatic patients. Psychotherapy and Psychosomatics, 22, 255-262.

Sifneos, P. E. (1996). Alexithymia: Past and present. The American Journal of Psychiatry, $153,137-142$.

Silvers, J. A., McRae, K., Gabrieli, J. D., Gross, J. J., Remy, K. A., \& Ochsner, K. N. (2012). Age-related differences in emotional reactivity, regulation, and rejection sensitivity in adolescence. Emotion, 12, 1235-1247.

Smith, R., Killgore, W. D. S., \& Lane, R. D. (2017). The structure of emotional experience and its relation to trait emotional awareness: A theoretical review. Emotion, 18, 670692.

Son, S., Jo, H., Rim, H. D., Kim, J. H., Kim, H.W., Bae, G. Y., \& Lee, S. J. (2012). A comparative study on alexithymia in depressive, somatoform, anxiety, and psychotic disorders among Koreans. Psychiatry Investigation, 9, 325-331.

Soto, J. A., Armenta, B. E., Perez, C. R., Zamboanga, B. L., Umaña-Taylor, A. J., Lee, R. M., ... \& Le, T. N. (2012). Strength in numbers? Cognitive reappraisal tendencies and 
psychological functioning among Latinos in the context of oppression. Cultural Diversity and Ethnic Minority Psychology, 18, 384-394.

Soto, J. A., Perez, C. R., Kim, Y. H., Lee, E. A., \& Minnick, M. R. (2011). Is expressive suppression always associated with poorer psychological functioning? A crosscultural comparison between European Americans and Hong Kong Chinese. Emotion, 11, 1450-1455.

Spaapen, D. L., Waters, F., Brummer, L., Stopa, L., \& Bucks, R. S. (2014). The Emotion Regulation Questionnaire: Validation of the ERQ-9 in two community samples. Psychological Assessment, 26, 1-7.

Spearman, C. (1904). "General Intelligence," objectively determined and measured. The American Journal of Psychology, 15, 201-292.

Spreen, O., \& Strauss, E. (2006). A compendium of neuropsychological tests: Administration, norms, and commentary. New York, NY, US: Oxford University Press.

Stanford Psychophysiology Laboratory (2018). Resources: The Emotion Regulation Questionnaire. Retrieved from https://spl.stanford.edu/resources

Stawarczyk, D., Majerus, S., Van der Linden, M., \& D'Argembeau, A. (2012). Using the daydreaming frequency scale to investigate the relationships between mindwandering, psychological well-being, and present-moment awareness. Frontiers in Psychology, 3, 1-15.

Stevens, J. P. (1992). Applied multivariate statistics for the social sciences. Hillsdale, NJ: Erlbaum. 
Strickland, J., Parry, C. L., Allan, M. M., \& Allan, A. (2017). Alexithymia among perpetrators of violent offences in Australia: Implications for Rehabilitation. Australian Psychologist, 52, 230-237.

Su, J. C., Lee, R. M., Park, I. J., Soto, J. A., Chang, J., Zamboanga, B. L., ... \& Seol, K. O. (2015). Differential links between expressive suppression and well-being among Chinese and Mexican American college students. Asian American Journal of Psychology, 6, 15-24.

Subic-Wrana, C., Bruder, S., Thomas, W., Lane, R. D., \& Köhle, K. (2005). Emotional awareness deficits in inpatients of a psychosomatic ward: A comparison of two different measures of alexithymia. Psychosomatic Medicine, 67, 483-489.

Suslow, T., \& Junghanns, K. (2002). Impairments of emotion situation priming in alexithymia. Personality and Individual Differences, 32, 541-550.

Svaldi, J., Griepenstroh, J., Tuschen-Caffier, B., \& Ehring, T. (2012). Emotion regulation deficits in eating disorders: A marker of eating pathology or general psychopathology? Psychiatry Research, 197, 103-111.

Swart, M., Kortekaas, R., \& Aleman, A. (2009). Dealing with feelings: Characterization of trait alexithymia on emotion regulation strategies and cognitive-emotional processing. PLoS One, 4, e5751.

Taylor, G. J., Bagby, R. M., \& Parker, J. D. (1992). The Revised Toronto Alexithymia Scale: Some reliability, validity, and normative data. Psychotherapy and Psychosomatics, $57,34-41$.

Taylor, G. J., Bagby, R. M., \& Parker, J. D. (1999). Disorders of affect regulation: Alexithymia in medical and psychiatric illness. UK: Cambridge University Press. 
Taylor, G. J., Bagby, R. M., \& Parker, J. D. (2003). The 20-item Toronto alexithymia scale: IV. Reliability and factorial validity in different languages and cultures. Journal of Psychosomatic Research, 55, 277-283.

Taylor, G. J., Bagby, R.M., \& Parker, J. D. (2016). What's in the name 'alexithymia'? A commentary on "Affective agnosia: Expansion of the alexithymia construct and a new opportunity to integrate and extend Freud's legacy". Neuroscience and Biobehavioral Reviews, 68, 1006-1020.

Taylor, G. J., Parker, J. D., Bagby, R. M., \& Bourke, M. P. (1996). Relationships between alexithymia and psychological characteristics associated with eating disorders. Journal of Psychosomatic Research, 41, 561-568.

Taylor, G. J., Ryan, D., \& Bagby, R. M. (1985). Toward the development of a new self report alexithymia scale. Psychotherapy and Psychosomatics, 44, 191-199.

Taylor, G. J.,\&Bagby, R.M. (2004). New trends in alexithymia research. Psychotherapy and Psychosomatics, 73, 68-77.

Telch, C. F., Agras, W. S., \& Linehan, M. M. (2001). Dialectical behavior therapy for binge eating disorder. Journal of Consulting and Clinical Psychology, 69, 1061-1065.

Thompson, R. A., \& Calkins, S. D. (1996). The double-edged sword: Emotional regulation for children at risk. Development and Psychopathology, 8, 163-182.

Thorberg, F. A., Young, R. M., Sullivan, K. A., \& Lyvers, M. (2009). Alexithymia and alcohol use disorders: A critical review. Addictive Behaviors, 34, 237-245.

Thorberg, F. A., Young, R. M., Sullivan, K. A., Lyvers, M., Hurst, C., Connor, J. P., \& Feeney, G. F. (2010). A confirmatory factor analysis of the Toronto alexithymia scale (TAS-20) in an alcohol-dependent sample. Psychiatry Research, 178, 565-567.

Thorndike, R. L. (1936). Factor analysis of social and abstract intelligence. Journal of Educational Psychology, 27, 231-233. 
Tsaousis, I., Taylor, G., Quilty, L., Georgiades, S., Stavrogiannopoulos, M., \& Bagby, R. M. (2010). Validation of a Greek adaptation of the 20-item Toronto alexithymia scale. Comprehensive Psychiatry, 51, 443-448.

van der Velde, J., Servaas, M. N., Goerlich, K. S., Bruggeman, R., Horton, P., Costafreda, S. G., \& Aleman, A. (2013). Neural correlates of alexithymia: A meta-analysis of emotion processing studies. Neuroscience and Biobehavioral Reviews, 37, 17741785.

van Dijke, A., Ford, J. D., van der Hart, O., van Son, M., van der Heijden, P., \& Bühring, M. (2010). Affect dysregulation in borderline personality disorder and somatoform disorder: Differentiating under-and over-regulation. Journal of Personality Disorders, 24, 296-311.

van Kleef, G. A., Homan, A. C., Beersma, B., \& van Knippenberg, D. (2010). On angry leaders and agreeable followers: How leaders' emotions and followers' personalities shape motivation and team performance. Psychological Science, 21, 1827-1834.

van Sonderen, E., Sanderman, R., \& Coyne, J. C. (2013). Ineffectiveness of reverse wording of questionnaire items: Let's learn from cows in the rain. PLoS One, 8, e68967.

Vermeulen, N., Luminet, O., \& Corneille, O. (2006). Alexithymia and the automatic processing of affective information: Evidence from the affective priming paradigm. Cognition and Emotion, 20, 64-91.

Vine, V., \& Aldao, A. (2014). Impaired emotional clarity and psychopathology: A transdiagnostic deficit with symptom-specific pathways through emotion regulation. Journal of Social and Clinical Psychology, 33, 319-342.

Vorst, H. C., \& Bermond, B. (2001). Validity and reliability of the Bermond-Vorst alexithymia questionnaire. Personality and Individual Differences, 30, 413-434. 
Waller, E., \& Scheidt, C. E. (2006). Somatoform disorders as disorders of affect regulation: a development perspective. International Review of Psychiatry, 18, 13-24.

Watters, C. A., Taylor, G. J., \& Bagby, R. M. (2016). Illuminating the theoretical components of alexithymia using bifactor modeling and network analysis. Psychological Assessment, 28, 627-638.

Watters, C. A., Taylor, G. J., Quilty, L. C., \& Bagby, R. M. (2016). An examination of the topology and measurement of the alexithymia construct using network analysis. Journal of Personality Assessment, 98, 649-659.

Watters, C., Taylor, G. J., Ayearst, L., \& Bagby, R. M. (2016). Measurement invariance of the English and French language versions of the 20-item Toronto alexithymia scale. European Journal of Psychological Assessment, 1-8. https://doi.org/10.1027/1015$5759 / \mathrm{a} 000365$.

Way, I. F., Applegate, B., Cai, X., Franck, L. K., Black-Pond, C., Yelsma, P., ... Muliett, M. (2010). Children's alexithymia measure (CAM): A new instrument for screening difficulties with emotional expression. Journal of Child and Adolescent Trauma, 3, 303-318.

Wechsler, D. (2008). Wechsler Adult Intelligence Scale-Fourth Edition: Technical and interpretive manual. San Antonio, TX: Pearson Assessment.

Weinberg, A., \& Klonsky, E. D. (2009). Measurement of emotion dysregulation in adolescents. Psychological Assessment, 21, 616-621.

Weiss, N. H., Gratz, K. L., \& Lavender, J. M. (2015). Factor structure and initial validation of a multidimensional measure of difficulties in the regulation of positive emotions: The DERS-positive. Behavior Modification, 39, 431-453.

Werner, K., \& Gross, J. J. (2010). Emotion regulation and psychopathology: A conceptual framework. In A. M. Kring, \& D. M. Sloan (Eds.). Emotion regulation and 
psychopathology: A transdiagnostic approach to etiology and treatment (pp. 13-37). New York, NY: Guilford.

Williams, C., \& Wood, R. L. (2010). Alexithymia and emotional empathy following traumatic brain injury. Journal of Clinical and Experimental Neuropsychology, 32, $259-267$.

Wiltink, J., Glaesmer, H., Canterino, M., Wolfling, K., Knebel, A., Kessler, H., . . B Buetel, M. E. (2011). Regulation of emotions in the community: Suppression and reappraisal strategies and its psychometric properties. Psycho-Social Medicine, 8, 1-12.

Zech, E., Luminet, O., Rimé, B., \& Wagner, H. (1999). Alexithymia and its measurement: Confirmatory factor analyses of the 20-item Toronto Alexithymia Scale and the Bermond-Vorst Alexithymia Questionnaire. European Journal of Personality, 13, $511-532$.

Zeitlin, S. B., \& McNally, R. J. (1993). Alexithymia and anxiety sensitivity in panic disorder and obsessive-compulsive disorder. The American Journal of Psychiatry, 150, 658660.

Zelkowitz, R. L., \& Cole, D. A. (2016). Measures of emotion reactivity and emotion regulation: Convergent and discriminant validity. Personality and Individual Differences, 102, 123-132.

Zelkowitz, R. L., Cole, D. A., Han, G. T., \& Tomarken, A. J. (2016). The incremental utility of emotion regulation but not emotion reactivity in nonsuicidal self injury. Suicide and Life-Threatening Behavior, 46, 545-562.

Zhu, X., Yi, J., Yao, S., Ryder, A. G., Taylor, G. J., \& Bagby, R. M. (2007). Cross-cultural validation of a Chinese translation of the 20 -item Toronto alexithymia scale. Comprehensive Psychiatry, 48, 489-496. 
Zimmermann, P. (1999). Structure and functions of internal working models of attachment and their role for emotion regulation. Attachment and Human Development, 1, 291306.

Zinbarg, R. E., \& Barlow, D. H. (1996). Structure of anxiety and the anxiety disorders: a hierarchical model. Journal of Abnormal Psychology, 105, 181-193.

Zou, C., Plaks, J. E., \& Peterson, J. B. (2017). Don't get too excited: Assessing individual differences in the down-regulation of positive emotions. Journal of Personality Assessment, 1-11. http://dx.doi.org/10.1080/00223891.2017.1339711. 
Table 1C

Pearson Bivariate Correlations between Scores on the TAS-20, BVAQ, DERS and PERS

\begin{tabular}{|c|c|c|c|c|c|c|c|c|c|c|c|c|c|c|}
\hline \multirow[b]{2}{*}{ Scale/subscale } & \multicolumn{4}{|c|}{ TAS-20 } & \multicolumn{7}{|c|}{ BVAQ } & \multicolumn{3}{|c|}{ DERS } \\
\hline & Total & DIF & DDF & EOT & $\begin{array}{c}\text { Cognitive } \\
\text { alexithymia }\end{array}$ & $\begin{array}{c}\text { Affective } \\
\text { alexithymia }\end{array}$ & $\begin{array}{c}\mathrm{D}- \\
\text { Verbalising }\end{array}$ & $\begin{array}{c}\mathrm{D}- \\
\text { Fantasising }\end{array}$ & $\begin{array}{c}\mathrm{D}- \\
\text { Identifying }\end{array}$ & $\begin{array}{c}\mathrm{D}- \\
\text { Emotionalising }\end{array}$ & $\begin{array}{c}\mathrm{D}- \\
\text { Analysing }\end{array}$ & $\begin{array}{c}\text { Alexithymia } \\
\text { composite }\end{array}$ & Clarity & Awareness \\
\hline \multicolumn{15}{|l|}{ TAS-20 } \\
\hline Total scale & - & $.84 * *$ & $.85^{* *}$ & $.70 * *$ & $.79 * *$ & -.06 & $.65^{* *}$ & -.08 & $.70^{* *}$ & .00 & $.56^{* *}$ & $.72 * *$ & $.72 * *$ & $.55^{* *}$ \\
\hline T-DIF & $.84 * *$ & - & $.58^{* *}$ & $.31 * *$ & $.54 * *$ & $-.22 * *$ & $.38 * *$ & $-.18^{* *}$ & $.65^{* * *}$ & $-.16^{*}$ & $.30 * *$ & $.53 * *$ & $.64 * *$ & $.30^{* *}$ \\
\hline T-DDF & $.85 * *$ & $.58 * *$ & - & $.45 * *$ & $.76 * *$ & -.07 & $.77 * *$ & $-.13 *$ & $.56^{* *}$ & .04 & $.44 * *$ & $.62 * *$ & $.63 * *$ & $.46^{* *}$ \\
\hline T-EOT & $.70 * *$ & $.31 * *$ & $.45^{* *}$ & - & $.62 * *$ & $.22 * *$ & $.43 * *$ & $.18^{*}$ & $.45^{* *}$ & $.16^{*}$ & $.65^{* *}$ & $.59 * *$ & $.40 * *$ & $.59 * *$ \\
\hline \multicolumn{15}{|l|}{ BVAQ } \\
\hline Cognitive alexithymia & $.79 * *$ & $.54 * *$ & $.76^{* *}$ & $.62 * *$ & - & $.17 *$ & $.84^{* *}$ & .06 & $.76^{* *}$ & $.23 * *$ & $.80 * *$ & $.78 * *$ & $.66 * *$ & $.69^{* *}$ \\
\hline Affective alexithymia & -.06 & $-.22 * *$ & -.07 & $.22 * *$ & $.17 *$ & - & .06 & $.81 * *$ & -.01 & $.72 * *$ & $.39 * *$ & .08 & $-.15^{*}$ & $.22 * *$ \\
\hline D-Verbalising & $.65 * *$ & $.38 * *$ & $.77 * *$ & $.43 * *$ & $.84^{* *}$ & .06 & - & -.03 & $.42 * *$ & $.14 *$ & $.48 * *$ & $.57 * *$ & $.48 * *$ & $.50 * *$ \\
\hline D-Fantasising & -.08 & $-.18 * *$ & $-.13 *$ & $.18 * *$ & .06 & $.81 * *$ & -.03 & - & -.02 & $.19^{* *}$ & $.21 * *$ & -.02 & $-.15^{*}$ & .09 \\
\hline D-Identifying & $.70^{* *}$ & $.65^{* *}$ & $.56^{* *}$ & $.45 * *$ & $.76 * *$ & -.01 & $.42 * *$ & -.02 & - & .00 & $.47 * *$ & $.66 * *$ & $.71 * *$ & $.47 * *$ \\
\hline D-Emotionalising & -.00 & $-.16^{*}$ & .04 & $.16^{*}$ & $.23 * *$ & $.72 * *$ & $.14 *$ & $.19^{* *}$ & .00 & - & $.41 * *$ & $.15^{*}$ & -.07 & $.28 * *$ \\
\hline D-Analysing & $.56^{* *}$ & $.30 * *$ & $.44 * *$ & $.65 * *$ & $.80^{* *}$ & $.39^{* *}$ & $.48 * *$ & $.21 * *$ & $.47 * *$ & $.41 * *$ & - & $.66^{* *}$ & $.41 * *$ & $.69^{* *}$ \\
\hline \multicolumn{15}{|l|}{ DERS } \\
\hline Alexithymia composite & $.72 * *$ & $.53 * *$ & $.62 * *$ & $.59 * *$ & $.78 * *$ & .08 & $.57 * *$ & -.02 & $.66^{* *}$ & $.15^{*}$ & $.66^{* *}$ & - & $.81^{* *}$ & $.91 * *$ \\
\hline Clarity & $.72 * *$ & $.66 * *$ & $.63 * *$ & $.40 * *$ & $.66 * *$ & $-.15^{*}$ & $.48^{* *}$ & $-.15^{*}$ & $.71 * *$ & -.07 & $.41 * *$ & $.81 * *$ & - & $.48^{* *}$ \\
\hline Awareness & $.55 * *$ & $.30 * *$ & $.46^{* *}$ & $.59 * *$ & $.69 * *$ & $.22 * *$ & $.50 * *$ & .09 & $.47 * *$ & $.28^{* *}$ & $.69 * *$ & $.91 * *$ & $.48 * *$ & - \\
\hline \multicolumn{15}{|l|}{ PERS } \\
\hline General positive reactivity & $-.29 * *$ & $-.23 * *$ & $-.26 * *$ & $-.20 * *$ & $-.36^{* *}$ & $-.19 * *$ & $-.30 * *$ & -.03 & $-.31 * *$ & $-.28 * *$ & $-.26 * *$ & $-.35 * *$ & $-.29 * *$ & $-.31 * *$ \\
\hline Positive-activation & $-.25 * *$ & $-.19 * *$ & $-.24 * *$ & $-.15^{*}$ & $-.33 * *$ & $-.18^{*}$ & $-.30 * *$ & -.05 & $-.28 * *$ & $-.24 * *$ & $-.21 * *$ & $-.30 * *$ & $-.24 * *$ & $-.26 * *$ \\
\hline Positive-intensity & -.08 & .01 & -.09 & $-.13^{*}$ & $-.16^{* *}$ & $-.26^{* *}$ & $-.17 *$ & -.10 & -.04 & $-.33 * *$ & $-.17^{*}$ & $-.17 *$ & -.07 & $-.21 * *$ \\
\hline Positive-duration & $-.40 * *$ & $-.38 * *$ & $-.32 * *$ & $-.23 * *$ & $-.41 * *$ & -.07 & $-.29 * *$ & .06 & $-.43 * *$ & $-.18 * *$ & $-.28 * *$ & $-.42 * *$ & $-.40 * *$ & $-.34 * *$ \\
\hline General negative reactivity & $.43 * *$ & $.53 * *$ & $.31^{* *}$ & $.14 *$ & $.29 * *$ & $-.34 * *$ & $.22 * *$ & $-.25 * *$ & $.38^{* *}$ & $-.28 * *$ & $.12 *$ & $.27 * *$ & $.43 * *$ & .09 \\
\hline Negative-activation & $.44 * *$ & $.52 * *$ & $.30 * *$ & $.19^{* *}$ & $.31 * *$ & $-.28 * *$ & $.21 * *$ & $-.18^{*}$ & $.39 * *$ & $-.27 * *$ & $.16^{*}$ & $.30 * *$ & $.42 * *$ & $.14 *$ \\
\hline Negative-intensity & $.32 * *$ & $.45^{* *}$ & $.24 * *$ & .00 & $.19 * *$ & $-.36^{* *}$ & $.15^{*}$ & $-.28 * *$ & $.29 * *$ & $-.27 * *$ & .02 & $.15^{*}$ & $.32 * *$ & -.01 \\
\hline Negative-duration & $.43 * *$ & $.50 * *$ & $.31 * *$ & $.18^{*}$ & $.31 * *$ & $-.32 * *$ & $.24 * *$ & $-.24 * *$ & $.38 * *$ & $-.25 * *$ & $.14^{*}$ & $.30 * *$ & $.45^{* *}$ & $.13^{*}$ \\
\hline
\end{tabular}

Note. $p<.001^{* *}, p<.05^{*}$. TAS-20 = Toronto Alexithymia Scale-20, BVAQ = Bermond-Vorst Alexithymia Questionnaire, DERS $=$ Difficulties in Emotion Regulation

Scale, PERS $=$ Perth Emotional Reactivity Scale. 